\title{
Correlation Transfer by Layer 5 Cortical Neurons Under Recreated Synaptic Inputs In Vitro
}

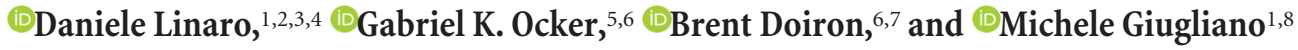 \\ ${ }^{1}$ Molecular, Cellular, and Network Excitability Laboratory, Department of Biomedical Sciences and Institute Born-Bunge, Universiteit Antwerpen, Wilrijk \\ B-2610, Belgium, ${ }^{2}$ Université Libre de Bruxelles (ULB), IRIBHM, and ULB Neuroscience Institute, Brussels B-1050, Belgium, ${ }^{3}$ VIB-KU Leuven Center for \\ Brain and Disease Research, Leuven B-3000, Belgium, ${ }^{4}$ Department of Neurosciences, Leuven Brain Institute, KU Leuven, Leuven B-3000, Belgium, \\ ${ }^{5}$ Department of Neuroscience, University of Pittsburgh, Pittsburgh, Pennsylvania 15260, ${ }^{6}$ Center for the Neural Basis of Cognition, University of Pittsburgh \\ and Carnegie Mellon University, Pittsburgh, Pennsylvania 15213, ${ }^{7}$ Department of Mathematics, University of Pittsburgh, Pittsburgh, Pennsylvania 15260, \\ and ${ }^{8}$ Scuola Internazionale Superiore di Studi Avanzati, Neuroscience Sector, Trieste, Italy I-34136
}

Correlated electrical activity in neurons is a prominent characteristic of cortical microcircuits. Despite a growing amount of evidence concerning both spike-count and subthreshold membrane potential pairwise correlations, little is known about how different types of cortical neurons convert correlated inputs into correlated outputs. We studied pyramidal neurons and two classes of GABAergic interneurons of layer 5 in neocortical brain slices obtained from rats of both sexes, and we stimulated them with biophysically realistic correlated inputs, generated using dynamic clamp. We found that the physiological differences between cell types manifested unique features in their capacity to transfer correlated inputs. We used linear response theory and computational modeling to gain clear insights into how cellular properties determine both the gain and timescale of correlation transfer, thus tying single-cell features with network interactions. Our results provide further ground for the functionally distinct roles played by various types of neuronal cells in the cortical microcircuit.

Key words: cortical interneurons; dynamic clamp; neocortex; noise correlations; pyramidal cells

\section{Significance Statement}

No matter how we probe the brain, we find correlated neuronal activity over a variety of spatial and temporal scales. For the cerebral cortex, significant evidence has accumulated on trial-to-trial covariability in synaptic inputs activation, subthreshold membrane potential fluctuations, and output spike trains. Although we do not yet fully understand their origin and whether they are detrimental or beneficial for information processing, we believe that clarifying how correlations emerge is pivotal for understanding large-scale neuronal network dynamics and computation. Here, we report quantitative differences between excitatory and inhibitory cells, as they relay input correlations into output correlations. We explain this heterogeneity by simple biophysical models and provide the most experimentally validated test of a theory for the emergence of correlations.

\section{Introduction}

Throughout the brain, the activity of neurons is correlated across multiple spatial and temporal scales (Cohen and Kohn, 2011;

\footnotetext{
Received Dec. 12, 2018; revised July 6, 2019; accepted July 12, 2019.

Author contributions: D.L., B.D., and M.G. designed research; D.L. and G.K.O. performed research; D.L. and M.G. wrote the first draft of the paper; D.L., G.K.O., B.D., and M.G. wrote the paper.

This work was supported by the European Union's Horizon 2020 Framework Programme for Research and Innovation (Grant \#785907, Human Brain Project SGA2), the Belgian Science Policy Office (Grant \#IUAP-VII/20), the Flemish Research Foundation (Grants \#G0F1517N and \#K201619N), and Scuola Internazionale Superiore di Studi Avanzati ("Collaborazione di Eccellenza 2018"), the National Institutes of Health (Grant \#1U19NS107613-01), the Vannevar Bush Faculty (Fellowship \#N00014-18-1-2002), and the Simons Foundation Collaboration on the Global Brain. The funders had no role in study design, data collection and analysis, decision to publish, or preparation of the manuscript. The data underlying these findings are fully available. Relevant datasets and analysis scripts have been stored at FigShare.com, including an index of the deposited data. We thankJ. Couto, A. Bacci, V. Bonin, and K. Farrow for discussions and to D. Van Dyck and M. Wijnants for excellent technical assistance.
}

Doiron et al., 2016). In the cortex, neurons embedded in the same microcircuit show a high degree of functional similarity, not only in the correlations between spike trains (deCharms and Merzenich, 1996; Bair et al., 2001; Erisken et al., 2014), but also in sub-threshold voltage fluctuations (Poulet and Petersen, 2008; Gentet et al., 2010, 2012). Several studies investigated the magnitude and timescales of spike-count correlations, known also as noise correlations (Cohen and Kohn, 2011). In primates, for example, correlations arise on relatively short timescales (i.e., up to a few hundred milliseconds) in the visual area MT (Bair

The authors declare no competing financial interests.

Correspondence should be addressed to Michele Giugliano at michele.giugliano@sissa.it.

https://doi.org/10.1523/JNEUROSCI.3169-18.2019

Copyright $\odot 2019$ the authors 
et al., 2001) and their magnitude is strongly modulated both by the stimulus properties and internal brain state (Steinmetz et al., 2000).

The cortical origin of spike-count correlations has been investigated extensively in vitro and in theoretical studies. By artificially constraining the fraction of common inputs received by pairs of neurons, de la Rocha et al. (2007) demonstrated experimentally that spike-count correlations between spike trains increase with the mean firing rate of the cell pair. Albeit general, this result was based on current-clamp stimuli, which only approximately approximate the summed activity of a large number of excitatory and inhibitory inputs. The in vitro and in silico study of (Litwin-Kumar et al., 2011) removed this limitation, considering the high-conductance state of intact cortical circuits (Destexhe et al., 2001, 2003). The high-conductance state transferred short time scale $(\sim 10 \mathrm{~ms})$ correlations better than the low-conductance state. However, the opposite was true for long time scale $(\sim 100 \mathrm{~ms})$, prompting the overall effect of a shift in background conductance to be termed "correlation shaping" (Litwin-Kumar et al., 2011). These theoretical results were further experimentally validated in vitro, although for only one value of the mean firing rate.

Theoretical arguments (de la Rocha et al., 2007; LitwinKumar et al., 2011; Doiron et al., 2016) explain these modulations in correlation transfer by associated shifts in the dynamical transfer functions of each cell in the pair (Köndgen et al., 2008; Linaro et al., 2018). This is consistent with studies showing that intrinsic properties-such as whether the neuron acts like an integrator or a coincidence detector (König et al., 1996) —also determine correlation transfer (Hong et al., 2012). It is well known that the input-output transfer and other intrinsic properties of neurons are quite distinct between cell classes (Connors and Gutnick, 1990; Markram et al., 2004). Nevertheless, despite this vast literature, little is known about the way in which different cell types transmit correlations.

Here, we present the results of dynamic-clamp experiments carried out in pyramidal cells and in fast-spiking (FS) and nonfast-spiking (NON-FS) interneurons from slices of rat cortical tissue. We found that, for low input correlation and across all cell types, the experimentally measured values of output covariance and their dependency on firing rate are in quantitative agreement with the values computed using linear response theory (LitwinKumar et al., 2011; Ocker and Doiron, 2014). Interestingly however, FS interneurons displayed significantly larger values of output covariance, making this cell type particularly suited for transmitting correlations to its postsynaptic targets. These results can be explained by considering single-cell properties-the steepness of the stationary rate-current curve, the membrane time constant and the degree of spike-frequency adaptationand how they are modulated by dynamic-clamp stimuli. Indeed, by using single-compartment integrate-and-fire models that recapitulate the electrophysiological response properties of the three cell types considered here, we could produce covariance values that qualitatively replicate our experimental observations. Additionally, we found that in pyramidal cells the correlationshaping mechanism introduced by (Litwin-Kumar et al., 2011) crucially depends on the properties of the stimulation paradigm.

In summary, our results highlight how the intrinsic properties of distinct neuronal types affect their correlated firing and hint at possible functionally distinct roles of different cell types in propagating correlations within cortical circuits.

\section{Materials and Methods}

Brain tissue slice preparation. Experiments were performed in accordance with international and institutional guidelines on animal welfare. All procedures were approved by the Ethical Committee of the Department of Biomedical Sciences of the University of Antwerp (permission no. 2011_87), and licensed by the Belgian Animal, Plant and Food Directorate-General of the Federal Department of Public Health, Safety of the Food Chain and the Environment (license no. LA1100469). Wistar rats of either sex (2-4 weeks old) were anesthetized using Isoflurane (IsoFlo, Abbott, USA) and decapitated. Brains were rapidly extracted and immersed, to be sliced, in ice-cold Artificial CSF (ACSF), containing (in $m M): 125 \mathrm{NaCl}, 25 \mathrm{NaHCO}_{3}, 2.5 \mathrm{KCl}, 1.25 \mathrm{NaH}_{2} \mathrm{PO}_{4}, 2 \mathrm{CaCl}_{2}, 1 \mathrm{MgCl}_{2}$, 25 glucose, saturated with $95 \% \mathrm{O}_{2}$ and $5 \% \mathrm{CO}_{2}$. Parasagittal sections (300 $\mu \mathrm{m}$ thick) of the primary somatosensory cortex were cut using a vibratome (VT1000 S, Leica Microsystems) and then incubated in ACSF at $36^{\circ} \mathrm{C}$ for at least $45 \mathrm{~min}$. Slices were then stored at room temperature, until transfer to the recording chamber.

An upright microscope (Leica Microsystems, DMLFS), equipped with infrared differential interference contrast videomicroscopy, was employed to visually identify layer 5 (L5) cortical neurons in a submerged slice recording chamber, under $60 \times$ magnification. Recordings were performed at $32 \pm 1^{\circ} \mathrm{C}$, under continuous perfusion with ACSF at a rate of $1 \mathrm{~mL} / \mathrm{min}$. All chemicals were obtained from Sigma-Aldrich.

Electrophysiological recordings. Patch-clamp recordings were obtained from the cell somata in the whole-cell configuration, employing glass pipettes pulled on a horizontal puller (P97, Sutter Instruments) from filamented borosilicate glass capillaries (Hilgenberg). Pipette electrode resistance was in the range 4-8 $M \Omega$, when filled with an intracellular solution containing the following (in $m M$ ): $115 \mathrm{~K}$-gluconate, $20 \mathrm{KCl}, 10$ HEPES, 4 Mg-ATP, $0.3 \mathrm{Na}_{2}$-GTP, $10 \mathrm{Na}_{2}$-phosphocreatine, $\mathrm{pH}$ adjusted to 7.3 with $\mathrm{KOH}$.

Recordings and current injections were performed with a single electrode by a current-clamp amplifier (EPC 10, HEKA Electronics), but neither the bridge balance nor capacitance neutralization circuitries of the amplifiers were activated during the experiments. Instead, signal transfer properties of the glass microelectrodes were repeatedly estimated, by a linear nonparametric identification method (Brette et al., 2008), throughout each recording session. Quantified as the impulse response, the microelectrode transfer properties were employed to compensate for artifacts in the recorded membrane potential offline or online (i.e., in conductance-clamp experiments). All experiments were performed using the software toolbox LCG (Linaro et al., 2014). Liquid junction potentials were left uncorrected, but are not expected to affect our conclusions. Signals were sampled at a rate of $20 \mathrm{kHz}$ and digitized at 16 bits, with an A/D conversion board (NI PCI6221 , National Instruments). The D/A converter of the same board was used to synthesize the external voltage-commands to the patch-clamp amplifier, at $20 \mathrm{kHz}$ and 16 bits of resolution.

Current-clamp and conductance-clamp stimulation waveforms. Our stimulation protocol closely follows the framework introduced in (de la Rocha et al., 2007), repeats it, and extends it to the case of conductance inputs, recreated by the dynamic-clamp technique (Robinson and Kawai, 1993; Chance et al., 2002) (see Fig. 3A). Although only one neuron was stimulated and recorded at a time, our entire set of experiments was examined a posteriori to study and quantify the similarity between the responses from pairs of (unconnected) neurons. In fact, synaptic inputs to these neuronal pairs had been synthesized a priori with a desired degree of similarity.

In the case of current-driven stimuli as in (de la Rocha et al., 2007), each of the currents $I_{1}(t)$ and $I_{1}(t)$ injected (nonsimultaneously) into two neurons was defined as the sum of an independent term and of a common term as follows:

$$
\begin{aligned}
& I_{1}(t)=\mu_{1}+\sigma_{1} \cdot\left(\sqrt{1-c} \cdot \eta_{1}(t)+\sqrt{c} \cdot \eta_{c}(t)\right) \\
& I_{2}(t)=\mu_{2}+\sigma_{2} \cdot\left(\sqrt{1-c} \cdot \eta_{2}(t)+\sqrt{c} \cdot \eta_{c}(t)\right)
\end{aligned}
$$

where $c, \mu_{1}, \sigma_{1}, \mu_{2}, \sigma_{2}$ are numerical parameters varied experimentally, and $\eta_{1}(t), \eta_{2}(t)$ and $\eta_{c}(t)$ are randomly fluctuating waveforms, generated offline as independent realizations of an Ornstein-Uhlenbeck stochastic 
process (Uhlenbeck and Ornstein, 1930; Cox and Miller, 1965). These waveforms have Gaussian amplitude distribution, zero mean, unitary variance, and an auto-correlation function exponentially decaying with time constant of $\tau=5 \mathrm{~ms}$. They are generated as discrete-time approximations, by independently iterating for each sampling interval $\Delta t$ (i.e., $\left.\Delta t=(20 \mathrm{kHz})^{-1}\right)$ the algebraic expressions $\eta_{x}(t+\Delta t)=(1-\Delta t / \tau) \cdot \eta_{x}(t)$ $+\sqrt{ } 2 \Delta t / \tau \cdot \xi_{x}(t)$ with $x \in\{1,2, c\}$, where the quantities $\xi_{1}(t), \xi_{2}(t)$, and $\xi_{c}(t)$ are three independent sequences of Gaussian numbers obtained by employing three distinct initial seeds for a pseudo-random number generator (Press, 2007). By such a construction, $I_{1}(t)$ and $I_{2}(t)$ are realizations of colored Gaussian stochastic processes, with means $\mu_{1}$ and $\mu_{2}$, standarddeviations $\sigma_{1}$ and $\sigma_{2}$, respectively, and identical autocorrelation length $\tau=5 \mathrm{~ms}$. Importantly, their cross-correlation coefficient $c$, which is under the direct control of the experimenter, determines a priori the degree of similarity between the waveforms and represents, in a compact form, both the fraction of common inputs and the synchronous inputs coming from distinct presynaptic sources $(0 \leq c \leq 1)$ (see Fig. 3B).

In the case of conductance-driven stimuli, the total currents $I_{1}(t)$ and $I_{2}(t)$ were computergenerated in real time by conductance-clamp (Destexhe and Paré, 1999; Destexhe et al., 2001; Chance et al., 2002; Linaro et al., 2014) as a barrage of excitatory and inhibitory synaptic conductance inputs:

$$
\begin{aligned}
I_{1}(t)=G_{E, 1}(t)\left(E_{E}-V_{1}(t)\right)+G_{I, 1}(t)\left(E_{I}\right. & \\
& \left.-V_{1}(t)\right) \\
I_{2}(t)=G_{E, 2}(t)\left(E_{E}-V_{2}(t)\right)+G_{I, 2}(t)\left(E_{I}\right. & \left.-V_{2}(t)\right)
\end{aligned}
$$

where $G_{E, 1}(t), G_{E, 2}(t)\left(G_{I, 1}(t), G_{I, 2}(t)\right)$ are randomly fluctuating excitatory (inhibitory) synthetic synaptic conductance waveforms, whose apparent reversal potential is chosen as $E_{E}=0 m V\left(E_{I}=-80 m V\right)$ (Chance et al., 2002), and where $V_{1}(t)$ and $V_{2}(t)$ are the membrane potentials instantaneously recorded from the two cells, as shown schematically in Fig. $3 A$.

Similarly to Equation 1 , the excitatory (inhibitory) conductance waveform $G_{E, 1}(t), G_{E, 2}(t)\left(G_{I, 1}(t), G_{I, 2}(t)\right)$ were defined by the sum of an independent term and of a common term as follows:

$$
\begin{aligned}
& G_{E, 1}(t)=\bar{g}_{E, 1}+\sigma_{E, 2} \cdot\left(\sqrt{1-c} \cdot \eta_{E, 1}(t)+\sqrt{c} \cdot \eta_{E, c}(t)\right) \\
& G_{E, 2}(t)=\bar{g}_{E, 2}+\sigma_{E, 2} \cdot\left(\sqrt{1-c} \cdot \eta_{E, 2}(t)+\sqrt{c} \cdot \eta_{E, c}(t)\right) \\
& G_{I, 1}(t)=\bar{g}_{I, 1}+\sigma_{I, 1} \cdot\left(\sqrt{1-c} \cdot \eta_{I, 1}(t)+\sqrt{c} \cdot \eta_{I, c}(t)\right) \\
& G_{I, 2}(t)=\bar{g}_{I, 2}+\sigma_{I, 2} \cdot\left(\sqrt{1-c} \cdot \eta_{I, 2}(t)+\sqrt{c} \cdot \eta_{I, c}(t)\right)
\end{aligned}
$$

where $\eta_{E, 1}(t), \eta_{E, 2}(t), \eta_{E, c}(t), \eta_{I, 1}(t), \eta_{I, 2}(t), \eta_{I, c}(t)$, are six independent realizations of an Ornstein-Uhlenbeck stochastic process, generated offline as already described. These processes have heterogeneous autocorrelation time lengths $\left(\tau_{E}=5 \mathrm{~ms}\right.$ and $\tau_{I}=10 \mathrm{~ms}$ for excitatory and inhibitory inputs, respectively), capturing the distinct decay kinetics of synaptic currents mediated by AMPA- and by GABA-A-receptors (Tuckwell, 1989). $\bar{g}_{E, 1}$ and $\bar{g}_{E, 2}\left(\bar{g}_{I, 1}\right.$ and $\left.\bar{g}_{I, 2}\right)$ and $\bar{\sigma}_{E, 1}$ and $\bar{\sigma}_{E, 2}\left(\bar{\sigma}_{I, 1}\right.$ and $\left.\bar{\sigma}_{I, 2}\right)$ represent the means and standard deviations of the total excitatory (inhibitory) synaptic conductances, for each of the cells in the pair. Under the diffusion approximation (Tuckwell, 1989), Eq. 3 mimics the collec- tive effect of the asynchronous activation of a large number of presynaptic excitatory (inhibitory) afferents, each with a corresponding a unitary excitatory (inhibitory) postsynaptic peak conductance $g_{E P S C}\left(g_{I P S C}\right)$. If the presynaptic mean frequency of excitatory (inhibitory) activation is indicated by $R_{E}\left(R_{I}\right)$, then $\left(\bar{g}_{E}\left(\bar{g}_{I}\right)\right.$ and $\left.\bar{\sigma}_{E}\left(\bar{\sigma}_{I}\right)\right)$ can be expressed as follows (Destexhe and Bal, 2009):

$$
\begin{array}{cc}
\bar{g}_{E}=g_{E P S C} \cdot \tau_{E} \cdot R_{E} & \sigma_{E}=\sqrt{0.5 \cdot g_{E P S C}{ }^{2} \cdot \tau_{E} \cdot R_{E}} \\
\bar{g}_{I}=g_{I P S C} \cdot \tau_{I} \cdot R_{I} & \sigma_{I}=\sqrt{0.5 \cdot g_{I P S C}{ }^{2} \cdot \tau_{I} \cdot R_{I}}
\end{array}
$$

In all our experiments, $g_{E P S C}$ was fixed to $2 \%$ of the inverse of the input resistance of the cell, while $g_{I P S C}$ was fixed to $6 \%$ of the same value, as proposed in (Chance et al., 2002). The mean firing rate of the presynaptic inhibitory population was fixed to $R_{I}=3000 \mathrm{~Hz}$, while $R_{E}$ was determined as the value balancing the mean excitatory and inhibitory drives, whenever the postsynaptic membrane potential fluctuates around a value $V_{0}$. By construction, at such a value $V_{0}$ of the membrane potential, the mean current injected into each neuron (Eq. 2) vanishes as follows:

$$
\begin{gathered}
0=\langle I(t)\rangle \approx \bar{g}_{E} \cdot\left(E_{E}-V_{0}\right)+\bar{g}_{I} \cdot\left(E_{I}-V_{0}\right) \\
\Leftrightarrow R_{E}=\left[g_{I P S C} \cdot \tau_{I} \cdot R_{I} \cdot\left(E_{I}-V_{0}\right)\right] /\left[g_{E P S C} \cdot \tau_{E} \cdot\left(E_{E}-V_{0}\right)\right]
\end{gathered}
$$

By this definition, acting on the value of a single parameter $V_{0}$ allows one to change the ratio between excitatory and inhibitory inputs and thus to 
A
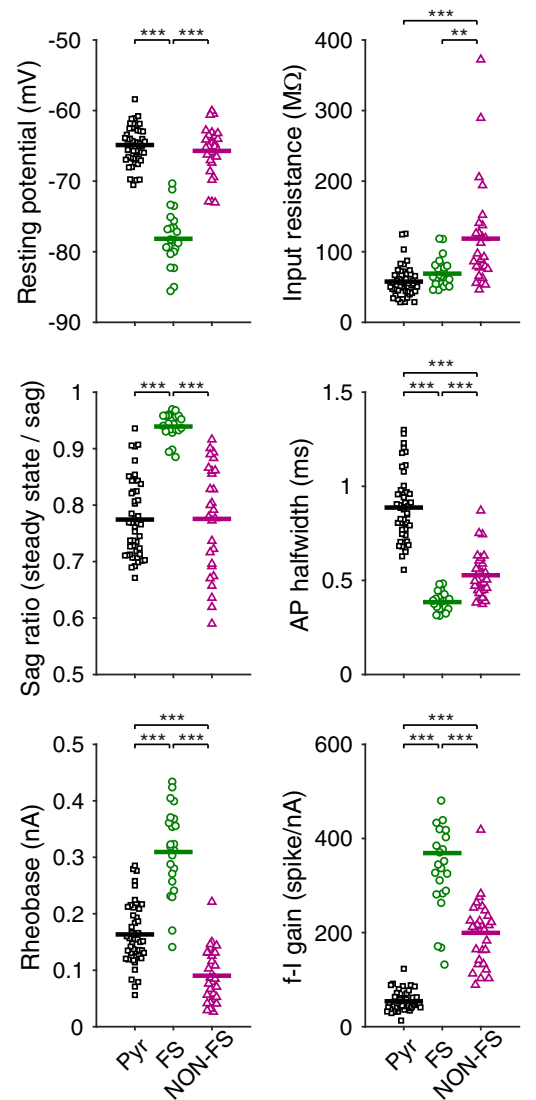
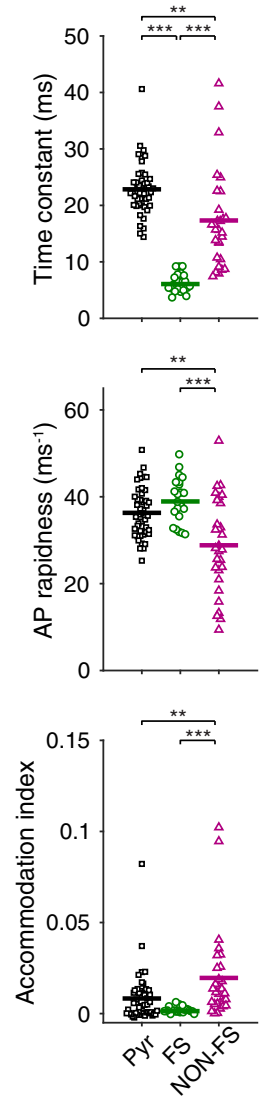

B

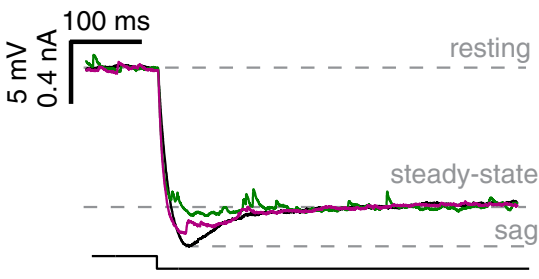

C

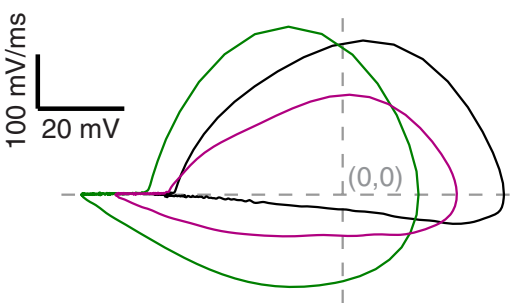

D

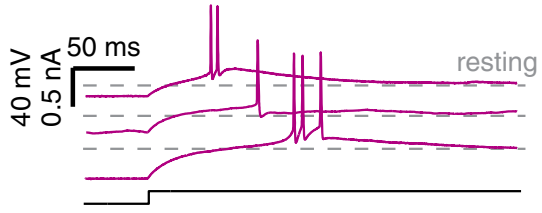

E

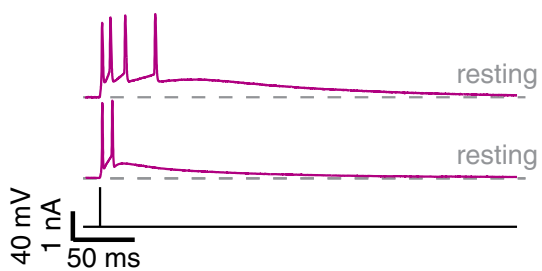

Figure 2. Distinct electrophysiological features of three cortical cell types. Our classification of pyramidal cells, FS and NON-FS interneurons is supported by a well-known heterogeneity of passive and active electrophysiological observables, as reported in the literature for basket cells and Martinotti cells and shown in $\boldsymbol{A}$ for our dataset. These observables were compared across 47 pyramidal cells, and $23 \mathrm{FS}$ and 29 NON-FS interneurons, and their statistically significant differences complement the diversity of transient and stationary firing transfer properties described in Figure 1. Two and three asterisks indicate a value of $p<0.01$ and $p<0.001$, respectively, for statistically significant differences as assessed by a Kruskal-Wallis test. $\boldsymbol{B}$, Representative voltage deflections in the three cell types in response to a step of current that caused a hyperpolarization of $\sim 10 \mathrm{mV}$. Note the comparable amount of sag in the response of the pyramidal cell and NON-FS interneuron, and the almost complete lack of sag in the response of the FS interneuron. The membrane voltages have been vertically aligned to the value of the resting potential before the stimulus onset, for ease of comparison. C, Representative voltage traces in the plane $d V / d t$ versus $V$ of the average action potential for the three cell types. Note how pyramidal cells display the largest action potentials, with onset speeds comparable to those of FS interneurons, which however are also characterized by an extremely fast AP offset. D, E, Rebound firing following hyperpolarization and burst generation in response to brief current pulses have been reported as distinctive features of somatostatin-positive NON-FS interneurons: these properties were observed in our experiments for NON-FS cells only, as shown here in five representative cells.

alter the output firing rate of the patched neuron. In analogy to the current-driven synaptic inputs (Eq. 1), where $\mu, \sigma$ were employed to increase or decrease the neuronal firing rate of each patched cell, $V_{0}$ was employed to study the dependence of the correlation transfer on the neuronal firing rate in the case of conductance injection.

All electrophysiological experiments were carried out with LCG (Linaro et al., 2014).

Experimental design. As in (de la Rocha et al., 2007), for each recorded neuron we delivered blocks of $N=100$ stimulation trials, each lasting $1.1 \mathrm{~s}$ and interleaved by interstimulus intervals lasting $1-6 s$, depending on the elicited firing rate (i.e., the higher the rate the longer the resting interval). Each block was repeated for 1-5 times, during which the parameters of the stimulation (e.g., $c$ and the pair $\mu, \sigma$ or the value of $V_{0}$ ) were kept constant. This series of repetitions was termed a stimulus set, and each neuron was presented with 1-7 distinct stimulus sets, differing in the values chosen for $c$ and $\mu, \sigma$, or for $c$ and $V_{0}$.

The identification of each patch electrode transfer properties, required for the Active Electrode Compensation, was performed once at the beginning of each block (i.e., every 5-10 min of recording), although in some instances it was repeated halfway through the block.

The initial seeds of the pseudo-random number generator, used for generating the shared component of each stimulus, were identical across the repetitions of the stimulation blocks and across cells. Within a block, each trial was generated by a distinct seed, so that a total of $N$ seeds for generating $\eta_{c}(t)$ in Eq. 1 and of $2 N$ seeds for generating $\eta_{E, c}(t), \eta_{I, c}(t)$ in
Eq. 3 were chosen once for all the experiments. Conversely, the seeds of all the independent components (i.e., $\eta_{1}(t), \eta_{2}(t)$ in Eq. $1 ; \eta_{E, 1}(t), \eta_{E, 2}(t)$, $\eta_{I, 1}(t), \eta_{I, 2}(t)$ in Eq. 3 ) were never fixed, but instead varied across trials, repetitions, and cells. All LCG scripts, configuration files, and command line strings required to precisely replicate our experimental protocols, together with the MATLAB scripts for the offline analysis of the recorded data, are available from FigShare.com.

After establishing the whole-cell configuration, in a subset of the experiments (see Figs. 1 and 5), we initially also probed the steady-state frequency-current curve of the neuron as in (Chance et al., 2002). Specifically, we injected $1 s$-long DC depolarizing current steps increasing amplitude, superimposed to conductance-driven recreated synaptic inputs, and measured the evoked firing rate as the number of emitted spikes divided by $0.9 s$, after discarding the first $0.1 s$ of the response to each stimulation step. Equation 3 was used to generate the synaptic inputs, with $c$ set to zero and $\eta_{E, 1}(t), \eta_{E, 2}(t), \eta_{I, 1}(t), \eta_{I, 2}(t)$ generated as independent realizations. The value of $V_{0}$ was fixed so that the neuron fired on the average at $\sim 0.5 \mathrm{~Hz}$, in the absence of any additional DC current step. This ensured that the modulation effect of the conductance-driven inputs was purely divisive for the frequency-current curve (Chance et al., 2002).

Data analysis: passive and active membrane electrical properties. Data were analyzed off line, using custom MATLAB scripts (The MathWorks). Passive membrane electrical properties, such as the input resistance and membrane time constant, were evaluated. The time 
A

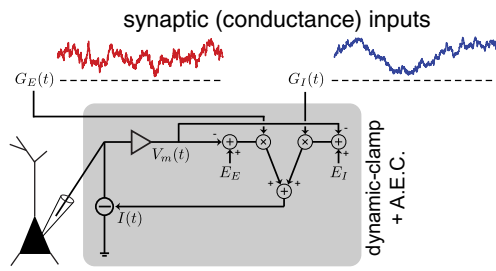

B

$c:$ fraction of common/shared synaptic inputs

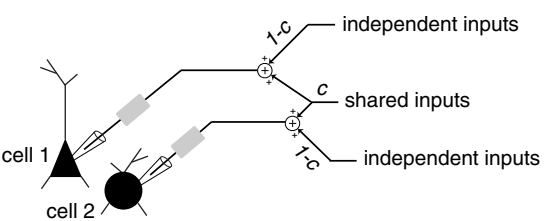

C

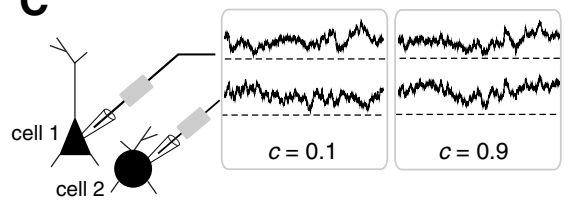

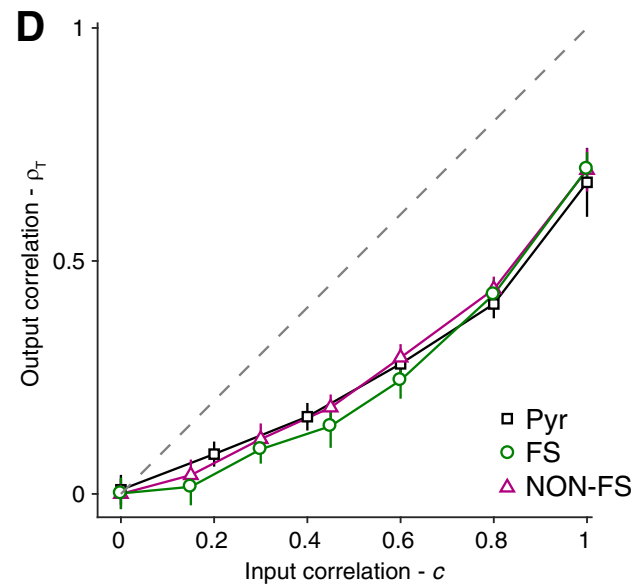

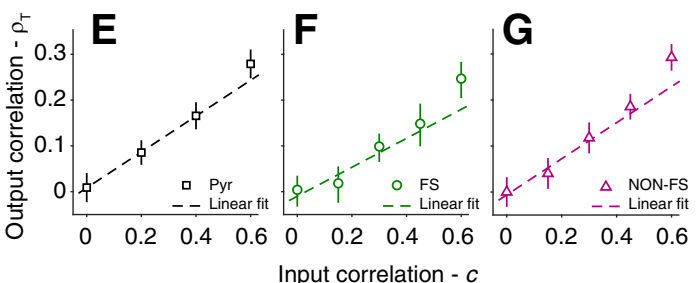

Figure 3. Output correlation $\rho_{\mathrm{T}}$ as a function of input correlation c, for three cortical cell types. Two sets of conductance-based fluctuating waveforms, sharing a common fraction c, were applied to pairs of anatomically unconnected neurons. At the steady-state, the correlation coefficient $\rho_{\mathrm{T}}$ between their spike responses was estimated over a spike-counting window of $T=40 \mathrm{~ms}$ and found to increase monotonically with increasing input correlation $c$, as intuitively expected. $A$, Schematic of the dynamic-clamp experimental setup: the total current injected into the cell is the sum of two conductance waveforms, one excitatory (red trace) and one inhibitory (blue trace), each multiplied by the appropriate driving force. This in turn depends on the instantaneous membrane potential, recorded in real-time by the system and filtered by the Active Electrode Compensation. $\boldsymbol{B}$, The stimulation to each cell in an unconnected pair comprises an independent and a shared component. The ratio of these two is regulated by the correlation coefficient $c$. C, Effect of varying con the conductance waveforms injected into the two cells. Notice the high (low) degree of similarity of the traces corresponding to $c=0.9(c=0.1)$. D. Mean values of $\rho_{\mathrm{T}}$ for $T=40 \mathrm{~ms}$ over the possible range of $\mathrm{c}$ for pyramidal cells (black markers), FS (green markers) and NON-FS interneurons (purple markers) cells. Error bars indicate the standard deviation. The dashed gray line has a unitary slope and highlights how the output correlation is always smaller than the input correlation. $\boldsymbol{E}$, For weak input correlations ( $c \leq 0.5$ ), the output correlation in pyramidal cells displayed a direct proportionality to $c$, a useful property for the subsequent theoretical interpretation of our results. Shown is the same data as in $\boldsymbol{D}$, magnified in the interval $c \in[0,0.6]$ and fit with a linear function of the form $\rho_{T}(c)=m c$, where $m$ is the proportionality coefficient. The fit was performed in the interval $c \in[0,0.4]$ to highlight how the value of $\rho_{\mathrm{T}}$ corresponding to $c=0.6$ departs from the linear relationship between input and output correlation. The data is the average of $n=6$ cells firing at a firing rate $v=$ $12.5 \pm 2.2$ spike/S. $\boldsymbol{F}$, Same as $\boldsymbol{E}$, but for FS interneurons $(N=5$ and $v=11 \pm 2.8$ spike/S). $\boldsymbol{G}$, Same as $\boldsymbol{E}$, but for NON-FS interneurons $(\boldsymbol{N}=6$ and $v=10 \pm 1.7$ spike/S).

constant was estimated as the longest time constant of a double exponential function, best fitted to the repolarizing phase following the application of a $10 \mathrm{~ms}$-long pulse of $-300 \mathrm{pA}$ amplitude. The input resistance was estimated as the slope of a steady-state current-voltage relationship, obtained by injecting 3-s-long subthreshold step current pulses with increasing amplitude. The rheobase current amplitude was estimated as the minimal DC stimulus amplitude necessary to elicit sustained firing.

The "sag" in the transient membrane potential trajectory in response to the injection of a $3 s$-long hyperpolarizing step of current, was quantified as the ratio $\Delta V_{\text {ss }} / \Delta V_{\text {peak }}$ : the steady-state voltage deflection $\Delta V_{\mathrm{ss}}$ was computed as the difference between $V_{\text {rest }}$, the resting potential averaged over the $500 \mathrm{~ms}$ preceding the step onset, and the (hyperpolarized) potential averaged in the final $500 \mathrm{~ms}$ of the step; $\Delta V_{\text {peak }}$ was computed as the difference between the above defined $V_{\text {rest }}$ and the minimum hyperpolarized potential, within the $300 \mathrm{~ms}$ following the onset of the step. The amplitude of the hyperpolarizing current was chosen such that $\Delta V_{\mathrm{ss}}$ was $\sim 10 \mathrm{mV}$.

The times of occurrence $\left\{t_{q}\right\}$ of the spikes fired by the neuron, in response to a given stimulation trial, were detected by finding the peaks of the intracellular voltage traces exceeding a threshold of $-20 \mathrm{mV}$. Other quantities were also extracted from each spike, such as (i) its threshold, defined as the voltage corresponding to the maximum of the third derivative of the membrane potential (Henze and Buzsáki, 2001; Kole and Stuart, 2008; Gentet et al., 2010); (ii) its amplitude, defined as the difference between its peak amplitude and the threshold; and (iii) its duration, measured as the width of the spike waveform at its half-amplitude.

To quantify the degree of spike frequency adaptation across cortical cell types, the accommodation index $A$ was also computed as follows (Shinomoto et al., 2003; Druckmann et al., 2007):

$$
A=\left(N_{s p k s}-4-1\right)^{-1} \cdot \sum_{q=4}^{N_{s p l s}-1}\left(\operatorname{ISI}_{q}-\operatorname{ISI}_{q-1}\right) /\left(\operatorname{ISI}_{q}+\operatorname{ISI}_{q-1}\right)
$$

where $N_{\text {spks }}$ is the number of spikes evoked by a constant depolarizing $1 s$-long step of current, ISI ${ }_{q}$ is the $q$-th interspike interval (i.e., ISI $_{q}=t_{q+1}-t_{q}$ ), and where the first four spikes were always discarded from the analysis (i.e., the sum starts from $q=4$ ). The amplitude of the step of current was chosen such that it elicited 15, 50 and 20 spikes/s for pyramidal, FS and NON-FS cells, respectively.

Experimental estimation of the spike-count covariance in neuronal pairs. For estimating the covariance of the spike counts, we closely followed the procedure outlined in (de la Rocha et al., 2007). In detail, under currentdriven and conductance-driven recreated synaptic inputs, and after discarding the first $0.1 s$ of each stimulation trial to minimize transient effects, we detected the spike times $\left\{t_{1}, t_{2}, \ldots, t_{q}, \ldots\right\}$ over the remaining interval of duration $L=1 s$. For each trial, the spike times were equivalently expressed as a discrete-time spike train, converting them into a binary string $y(j), j=\left[0,1,2, \ldots, \frac{L}{\Delta t}\right]$ upon uniform discretization of time, with a step of $\Delta t=1.2 \mathrm{~ms}$, where $\lfloor\mathrm{x}\rfloor$ indicates the largest integer not greater than $x$ :

$$
y(j)=\left\{\begin{array}{lc}
1 & \text { if there exists (a spike) } q: t_{q} \in[j \cdot \Delta t ;(j+1) \cdot \Delta t) \\
0 & \text { otherwise }
\end{array}\right.
$$

The discrete-time spike train $y(j)$ was then used to estimate the spike count, computed over a sliding window of duration $T$ (Dayan and Abbott, 2001): 


$$
n(j)=\sum_{i=j}^{j+\lfloor T / \Delta t\rfloor} y(i) \text { with } j=\{0,1,2, \ldots, M\}
$$

where $M=(L-T) / \Delta t$.

The spike count was obtained for every trial in a block of $N$ stimulations, for every block out of $R$ repetitions, and for every cell. In the following, we use the notation $n_{1}^{k, r}(j)$ and $n_{2}^{k, r^{\prime}}(j)$ to indicate, for two neurons, the spike counts of the response of cell 1 and cell 2, computed for the $k$-th trial and the $r$-th (or $r^{\prime}$-th) repetition (i.e., as the number of repetitions $R_{1}$ and $R_{2}$ may be different across cells). As in (de la Rocha et al., 2007), the estimate of the covariance of the spike counts of the cell pair, obtained during the corresponding trial, was corrected for the time-shift and averaged across all $R_{1}$ and $R_{2}$ repetitions and $N$ trials ${ }^{\star}$ as follows:

$$
\begin{aligned}
\operatorname{Cov}\left(n_{1}, n_{2}\right)=\frac{1}{N \cdot R_{1} \cdot R_{2}} \sum_{k, r, r^{\prime}}^{N, R_{1}, R_{2}} \frac{\left(\sum_{j=0}^{M} n_{1}^{k, r}(j) \cdot n_{2}^{k, r^{\prime}}(j)\right)}{(M+1)} & \\
& -\frac{\left(\sum_{j=0}^{M} n_{1}^{k, r}(j) \cdot n_{2}^{k+1, r^{\prime}}(j)\right)}{(M+1)},
\end{aligned}
$$

Finally, the correlation coefficient $\rho_{T}$ between the response of two neurons was obtained from the spike-count covariance as follows:

$$
\rho_{T}=\frac{\operatorname{Cov}\left(n_{1}, n_{2}\right)}{\sqrt{\operatorname{Var}\left(n_{1}\right) \operatorname{Var}\left(n_{2}\right)}},
$$

with $\operatorname{Var}\left(n_{i}\right)=\operatorname{Cov}\left(n_{i}, n_{i}\right)$. Throughout our work, we used the spikecount covariance, except for section "Output correlation depends on the fraction of common inputs" (see Fig. 3), where the linear relationship between input and output correlations is discussed.

Finally, as already mentioned in the previous sections, each cell was presented with several stimulus sets, each characterized by distinct values for $c$ and $\mu, \sigma$ or $c$ and $V_{0}$. As those parameters affect the mean spiking rate of the neurons, care was required to choose comparable responses across cells, out of the entire experimental dataset. Therefore, we computed the correlation coefficient only for those stimulus-set pairs $l, m$ that elicited firing rates $\nu_{1}$ and $\nu_{2}$ (estimated over the whole stimulus set) that did not differ by $>50 \%$, i.e., $0.5 \leq \nu_{1}^{l} / \nu_{2}^{m} \leq 2$. We also tested more stringent conditions on the ratio $\nu_{1}^{l} / \nu_{2}^{m}$ to select the stimulus-set pairs to include in the analysis (up to $0.8 \leq \nu_{1}^{l} / \nu_{2}^{m} \leq 1.25$ ) and found that this does not alter qualitatively the dependence of the covariance on the geometric mean firing rate of the cell pair.

Statistical analysis. Unless otherwise noted, all data are presented as mean \pm standard deviation and statistical significance was assessed using a two-sample Kolmogorov-Smirnov test (Press, 2007).

Theoretical prediction of the covariance values. We compared the empirical spike-count covariance to the values obtained from a theoretical prediction. This prediction is based on a well-established linear response theory for spike train covariability (de la Rocha et al., 2007). We first indicate in continuous time the spike train $y(t)$ as a sum of Dirac's Delta functions,

$$
y(t)=\sum_{q} \delta\left(t-t_{q}\right)
$$

and the corresponding spike count $n(t)$ as its convolution with the window function $w_{T}(t)$,

$$
n(t)=\int_{t}^{t+T} y(x) d x=y(t) * w_{T}(t),
$$

where $w_{T}(t)=1$, for $t$ and otherwise zero. The cross-covariance function of the spike counts of the two neurons receiving common input can be expressed in terms of the cross-covariance function of the spike trains (Cox and Isham, 1980) as

$$
\operatorname{Cov}\left(n_{1}, n_{2}\right)(\tau)=\operatorname{Cov}\left(y_{1}, y_{2}\right)(\tau) * \Delta_{T}(\tau)
$$

where $\Delta_{T}(\tau)$ is an even function defined as $\Delta_{T}(\tau)=\int_{-\infty}^{+\infty} w_{T}(s) w_{T}$ $(\tau+s) d s$ taking the value of $T-|\tau|$, for $\tau \in[-T$; $T]$, and otherwise zero.

By the Wiener-Khinchin theorem, the cross-covariance function $\operatorname{Cov}\left(y_{1}, y_{2}\right)(\tau)$ is the Fourier anti-transform of the cross-spectrum of the spike trains $\widehat{\operatorname{Cov}}\left(\hat{Y}_{1}, \hat{Y}_{2}\right)(\omega)$ as follows:

$$
\operatorname{Cov}\left(y_{1}, y_{2}\right)(\tau)=\frac{1}{2 \pi} \cdot \int_{-\infty}^{+\infty} \widehat{\operatorname{Cov}}\left(\hat{\mathrm{Y}}_{1}, \hat{\mathrm{Y}}_{2}\right)(\omega) \mathrm{e}^{j \omega \tau} \mathrm{d} \omega
$$

Considering current-clamp stimuli as in de la Rocha et al. (2007), and assuming a small amplitude for the common input $q(t)$ (i.e., $\sigma \cdot \sqrt{ } c$ ), we approximate the instantaneous spiking responses of the neurons as dominated by linear dynamical transfer properties. In the Fourier domain this corresponds to the following (Brunel et al., 2001):

$$
\begin{aligned}
& \hat{Y}_{1}(\omega) \cong \hat{Y}_{1,0}(\omega)+\hat{A}_{1}(\omega) \cdot \hat{Q}(\omega) \\
& \hat{Y}_{2}(\omega) \cong \hat{Y}_{2,0}(\omega)+\hat{A}_{2}(\omega) \cdot \hat{Q}(\omega)
\end{aligned}
$$

where $\hat{Q}(\omega)$ is the Fourier transform of $q(t), \hat{Y}_{i, 0}(\omega)$ corresponds to the baseline spike train (when $q(t)=0, \mathrm{i}=1,2$ ), and where $\hat{A}_{1}(\omega)$ and $\hat{A}_{2}(\omega)$ are the dynamical response functions of the two neurons. For these quantities, a full experimental characterization was demonstrated previously (Köndgen et al., 2008; Higgs and Spain, 2009; Ilin et al., 2013; Linaro et al., 2018).

Assuming that the two neurons' spike trains are conditionally independent, given the common input $q(t)$, we evaluate (Holden, 1976) and approximate the cross-spectrum by Eq. 15 and obtain

$$
\widehat{\operatorname{Cov}}\left(\hat{\mathrm{Y}}_{1}, \hat{\mathrm{Y}}_{1}\right)(\omega)=\left\langle\hat{\mathrm{Y}}_{1}^{*}(\omega) \cdot \hat{\mathrm{Y}}_{2}(\omega)\right\rangle \cong \hat{\mathrm{A}}_{1}^{*}(\omega) \cdot \hat{\mathrm{A}}_{2}(\omega) \cdot \hat{\mathrm{S}}(\omega)
$$

where $\hat{A}_{1}^{*}(\omega)$ is the complex conjugate of $\hat{A}_{1}^{*}(\omega)$, and $\hat{S}(\omega)=\hat{Q}^{*}(\omega) \cdot \hat{Q}(\omega)$ is the power spectrum of the common input $q(t)$.

Finally, estimating the covariance of the spike counts $n_{1}(t)$ and $n_{2}(t)$, requires evaluating Equation 13 in $\tau=0$ and substituting Equation 16 into Equation 14 as follows:

$$
\operatorname{Cov}\left(n_{1}, n_{2}\right)(0) \cong \frac{1}{2 \pi} \cdot \int_{-\infty}^{+\infty} \hat{\mathrm{A}}_{1}^{*}(\omega) \cdot \hat{\mathrm{A}}_{2}(\omega) \cdot \hat{\mathrm{S}}(\omega) \cdot \widehat{\Delta_{T}}(\omega) \mathrm{d} \omega
$$

where $\widehat{\Delta_{T}}(\omega)$ is the Fourier transform of $\Delta_{T}(\tau)$, and takes the form of $\widehat{\Delta_{T}}(\omega)=T^{2} \sin ^{2}(\omega T / 2) /(\omega T / 2)^{2}$. For large values of $T, \widehat{\Delta_{T}}(\omega)$ approaches a Dirac's Delta function $\widehat{\Delta_{T}}(\omega) \approx 2 \pi T \cdot \delta(\omega)$, thereby canceling the contribution to the integral for values of the integrand far from $\omega \approx 0$. In addition, the dynamical response of cortical cells is rather constant at low values of the Fourier frequencies (Fourcaud and Brunel, 2002; Köndgen et al., 2008; Testa-Silva et al., 2014; Linaro et al., 2018). For these two reasons, $\hat{A}_{1}(\omega)$ and $\hat{A}_{2}(\omega)$ are further approximated by their value in $\omega \approx 0$, which takes the form of the local slope (i.e., the steady-state gain) of the stationary rate-current curve $F(I)$, computed at the cell's mean output firing rate $\nu$ (Brunel et al., 2001; Chance et al., 2002).

$$
\left.\left.\operatorname{Cov}\left(n_{1}, n_{2}\right)(0) \approx \frac{d F_{1}(I)}{d I}\right|_{\nu_{1}} \cdot \frac{d F_{2}(I)}{d I}\right|_{\nu_{2}} \cdot \frac{1}{2 \pi} \cdot \int_{-\infty}^{+\infty} \hat{\mathrm{S}}(\omega) \cdot \Delta_{T}(\omega) \mathrm{d} \omega
$$

The covariance is therefore expected to be proportional to the product of the slopes of the rate-current curves of the two cells. For some experiments, we thus probed the rate-current curve of each neuron, in addition to the other stimulation protocol, and we employed its local slope to

*In order to make the notation consistent, it is intended that across the sum over $k$, there is one trial after the last which equals the first, i.e. $n_{2}^{M+1, r^{\prime}}=n_{2}^{1, r^{\prime}}$. 
approximate the neuron linear response function at the low frequencies needed to estimate spike-count covariances over long windows. In fact, although fast experimental techniques for the characterization of $\hat{A}_{1}(\omega)$ and $\hat{A}_{2}(\omega)$ are available (Higgs and Spain, 2009; Ilin et al., 2013), using the full transfer function is anyway not practical: $\hat{A}_{1}(\omega)$ and $\hat{A}_{2}(\omega)$ are in fact modulated by the mean firing rate $\nu_{1}$ and $\nu_{2}$ of the neurons (Linaro et al., 2018) and their complete estimate, across a sufficiently wide range of firing rates, is incompatible with the limited duration of each experiment.

Neuron models. For modeling different cortical cell types, we used an adaptive exponential integrate-and-fire model neuron (Brette and Gerstner, 2005), modified to incorporate a slow voltage-gated adaptation current $I_{\text {slow }}$. The neuron subthreshold membrane potential $V$ evolves in time as

$$
\begin{gathered}
\mathrm{C} \frac{d \mathrm{~V}}{d t}=\left[\bar{g} \cdot\left(V_{L}-V\right)+\bar{g} \cdot \Delta \cdot e^{\left(V-V_{T}\right) / \Delta}\right]+I_{\text {slow }}+I_{\text {ext }} \\
I_{\text {slow }}=\bar{g}_{w} \cdot w \cdot\left(V_{w}-V\right) \quad \tau_{w} \frac{d w}{d t}=\left(1+e^{-\left(V-V_{w h}\right) / D_{w}}\right)^{-1} \\
I_{\text {ext }}=G_{E}(t)\left(E_{E}-V\right)+G_{I}(t)\left(E_{I}-V\right)
\end{gathered}
$$

where $C$ and $\bar{g}$ are the capacitance and conductance per unit of membrane surface, respectively, $V_{L}$ the resting potential, $\Delta$ the spike steepness and $V_{T}$ the spike initiation threshold (Fourcaud and Brunel, 2002). $g_{w}$ represents the peak adaptation conductance, $w$ its activation state variable and $V_{w}$ its reversal potential. The steady-state activation of the adaptation conductance is a logistic function, monotonically increasing with the membrane potential, with half-maximal activation at $V_{w h}$ and activation slope $D_{w}$. As soon as $V$ crosses the peak threshold $V_{t h}$, it repolarizes linearly to its resting value $V_{L}$, over a (refractory) period $\tau_{\text {ref }}$.

To capture the features of each cortical cell type, we adapted the model parameter to reproduce the resting membrane potential, the accommodation index, the rheobase, and the slope of the rate-current relationship measured experimentally. We specifically adjusted the values of $V_{L}, C$ and $\bar{g}$ to match the resting membrane potential and membrane time constant for each neuron type. The spike initiation threshold of the model, $V_{L}$, was adjusted to ensure that the models had the same ordering of rheobases as in the experiments (i.e., NON-FS $<\mathrm{Pyr}<\mathrm{FS}$ ). Although the resulting ordering of $V_{T}$ was NON-FS $<$ FS $<$ Pyr, the value $\bar{g}_{w}$ of the adaptation conductance and the leak reversal potential also affect the rheobase.

The peak adaptation conductance, $g_{w}$, was then chosen to approximately match the accommodation indices for each neuron type, upon injecting a depolarizing constant current as in the experiments. All the parameters were identical across cell types for the three distinct model cells, as summarized in Table 1.

With the aim of reproducing the conductance-clamp protocols, the external current density $I_{\text {ext }}$ was synthesized as in the experiments by Eq. 2. For simplicity however, we set $g_{E P S C}=g_{I P S C}=0.06 \mathrm{mS} / \mathrm{cm}^{2}$, and considered instantaneous synaptic coupling (i.e., $\tau_{E}, \tau_{I} \rightarrow 0$ ) (Tuckwell, 1989 ), and $R_{I}=10 \mathrm{kHz}$, while changing the value of $R_{E}$ to change the output firing rates. Such a choice for $R_{I}$, used for (see Figure 8, C to $H$, reflects the firing rate of the summed activity of the modeled presynaptic inhibitory neurons that is proportional, for large populations, to the (sample) mean of a neuron's presynaptic inhibitory pool. Populationaveraged firing rates in rat cortex in vivo have been reported on the order of $\sim 1-5 \mathrm{~Hz}$, with fast-spiking putative inhibitory neurons firing faster than excitatory cells (e.g., Hengen et al., 2013; Buzsáki and Mizuseki, 2014). Within a thalamocortical projection column of the rat barrel cortex, there are $\sim 20^{\prime} 000$ neurons (Meyer et al., 2010). If $15 \%$ of those are inhibitory and their average connection probability is $1 / 3$, the mean number of partners would be 1000 , so that an average firing rate of $10 \mathrm{~Hz}$ per cell would yield a total inhibitory input rate of $10 \mathrm{kHz}$ for the entire inhibitory pool. In Figure $8 C$, we set a low excitatory rate of $1.75 \mathrm{kHz}$ to explore the static $F-I$ curve, while we varied the excitatory input rate in Figure $8, D$ to $H$.

Defining $\bar{g}_{\text {eff }}=\bar{g}+\bar{g}_{E}+\bar{g}_{I}$ and $V_{\text {eff }}=\bar{g}_{\text {eff }}^{-1} \cdot\left(\bar{g} \cdot V_{L}+\bar{g}_{E} \cdot E_{E}+\bar{g}_{I} \cdot E_{I}\right)$ as effective membrane conductance and effective resting potential, respectively, eq. 19 can be equivalently rewritten as
Table 1. Numerical parameters used in the integrate-and-fire model neurons

\begin{tabular}{llccc}
\hline Parameter & Description & Pyramidal & FS & NON-FS \\
\hline$C$ & Membrane capacitance & $1 \mu \mathrm{F} / \mathrm{cm}^{2}$ & $1 \mu \mathrm{F} / \mathrm{cm}^{2}$ & $1 \mu \mathrm{F} / \mathrm{cm}^{2}$ \\
$\tau$ & Passive membrane time constant & $25 \mathrm{~ms}$ & $15 \mathrm{~ms}$ & $25 \mathrm{~ms}$ \\
$V_{\mathrm{L}}$ & Leak reversal potential & $-65 \mathrm{mV}$ & $-80 \mathrm{mV}$ & $-65 \mathrm{mV}$ \\
$g_{\mathrm{w}}$ & Peak Kv7 conductance & $0.002 \mathrm{mS} / \mathrm{cm}^{2}$ & $0 \mathrm{mS} / \mathrm{cm}^{2}$ & $0.1 \mathrm{mS} / \mathrm{cm}^{2}$ \\
$\tau_{\mathrm{w}}$ & Kv7 activation time constant & $200 \mathrm{~ms}$ & $200 \mathrm{~ms}$ & $200 \mathrm{~ms}$ \\
$V_{\text {wh }}$ & Kv7 half-activation voltage & $-40 \mathrm{mV}$ & $-40 \mathrm{mV}$ & $-40 \mathrm{mV}$ \\
$D_{\mathrm{w}}$ & Slope of Kv7 activation & $8 \mathrm{mV}$ & $8 \mathrm{mV}$ & $8 \mathrm{mV}$ \\
$V_{\mathrm{w}}$ & Kv7 reversal potential & $-85 \mathrm{mV}$ & $-85 \mathrm{mV}$ & $-85 \mathrm{mV}$ \\
$\Delta$ & AP steepness & $1.4 \mathrm{mV}$ & $0.2 \mathrm{mV}$ & $1.4 \mathrm{mV}$ \\
$V_{T}$ & AP initiation threshold & $-46 \mathrm{mV}$ & $-50 \mathrm{mV}$ & $-52 \mathrm{mV}$ \\
$V_{\text {th }}$ & AP threshold & $0 \mathrm{mV}$ & $0 \mathrm{mV}$ & $0 \mathrm{mV}$ \\
$\tau_{\text {ref }}$ & Absolute refractory period & $6 \mathrm{~ms}$ & $1 \mathrm{~ms}$ & $4 \mathrm{~ms}$ \\
\hline
\end{tabular}

$$
\mathrm{C} \frac{d \mathrm{~V}}{d t}=\left[\bar{g}_{\text {eff }} \cdot\left(V_{\text {eff }}-V\right)+\bar{g} \cdot \Delta \cdot \mathrm{e}^{-\left(V-V_{T}\right) / \Delta}\right]+I_{\text {slow }}+i_{\text {ext }}
$$

$$
\begin{gathered}
I_{\text {slow }}=\bar{g}_{w} \cdot w \cdot\left(V_{w}-V\right) \quad \tau_{w} \frac{d w}{d t}=\left(1+\mathrm{e}^{-\left(V-V_{w h}\right) / D_{w}}\right)^{-1} \\
i_{\text {ext }}=g_{E}(t)\left(E_{E}-V\right)+g_{I}(t)\left(E_{I}-V\right)
\end{gathered}
$$

where $g_{E}(t)$ and $g_{I}(t)$ are the randomly fluctuating components of the synaptic conductances, with zero mean and with Dirac's Delta autocorrelation function.

For our numerical simulations as well as theoretical analysis of the correlation transfer in the model, we set $V=V_{\text {eff }}$ in the expression of $i_{\text {ext }}$ ignoring the multiplicative nature of the random fluctuations, which then took the approximate form as follows:

$$
i_{\text {ext }} \approx\left[\sigma_{E} \cdot\left(E_{E}-V_{e f f}\right)+\sigma_{I} \cdot\left(E_{I}-V_{e f f}\right)\right] \cdot \gamma(t)
$$

Under such an approximation, the dynamical response functions $\hat{A}(\omega)$ of the model neuron can be obtained directly, by numerical solution of the Fokker-Planck equation associated with Eqs. 20-21, as described in detail in (Richardson, 2009; Ocker and Doiron, 2014).

Similarly to the experiments, for a pair of neurons receiving partially correlated inputs, $\gamma(t)$ was chosen as follows:

$$
\begin{aligned}
& \gamma_{1}(t)=\sqrt{1-c} \cdot \eta_{1}(t)+\sqrt{c} \cdot \eta_{c}(t) \\
& \gamma_{2}(t)=\sqrt{1-c} \cdot \eta_{2}(t)+\sqrt{c} \cdot \eta_{c}(t)
\end{aligned}
$$

where $\eta_{1}(t), \eta_{2}(t)$ and $\eta_{c}(t)$ are randomly fluctuating waveforms, generated as independent realizations of a Gaussian white noise, with zero mean and unitary variance, instead of an Ornstein-Uhlenbeck process to facilitate the mathematical analysis by Fokker-Planck equation (Brunel et al., 2001; Fourcaud and Brunel, 2002).

For small values of $c$, eq. 18 could then be used instead of Eq. 17 to calculate the covariance of spike counts in the two model neurons. The MATLAB scripts for reproducing Figure 8 are available on FigShare.com.

\section{Results}

We closely followed the approach of de la Rocha et al. (2007) and extended it by dynamic-clamp to the case of conductance inputs, in addition to conventional current inputs. We examined in the details how altering the fraction of common inputs changes the similarity of their output spike trains, in pairs of unconnected neurons.

\section{Cell types and electrophysiological responses}

We recorded in vitro from $n=47$ pyramidal cells and $n=52$ interneurons in L5 of the rat primary somatosensory cortex, during the application of current- and conductance-clamp stimulation. Pyramidal cells were readily identified by the presence of a 

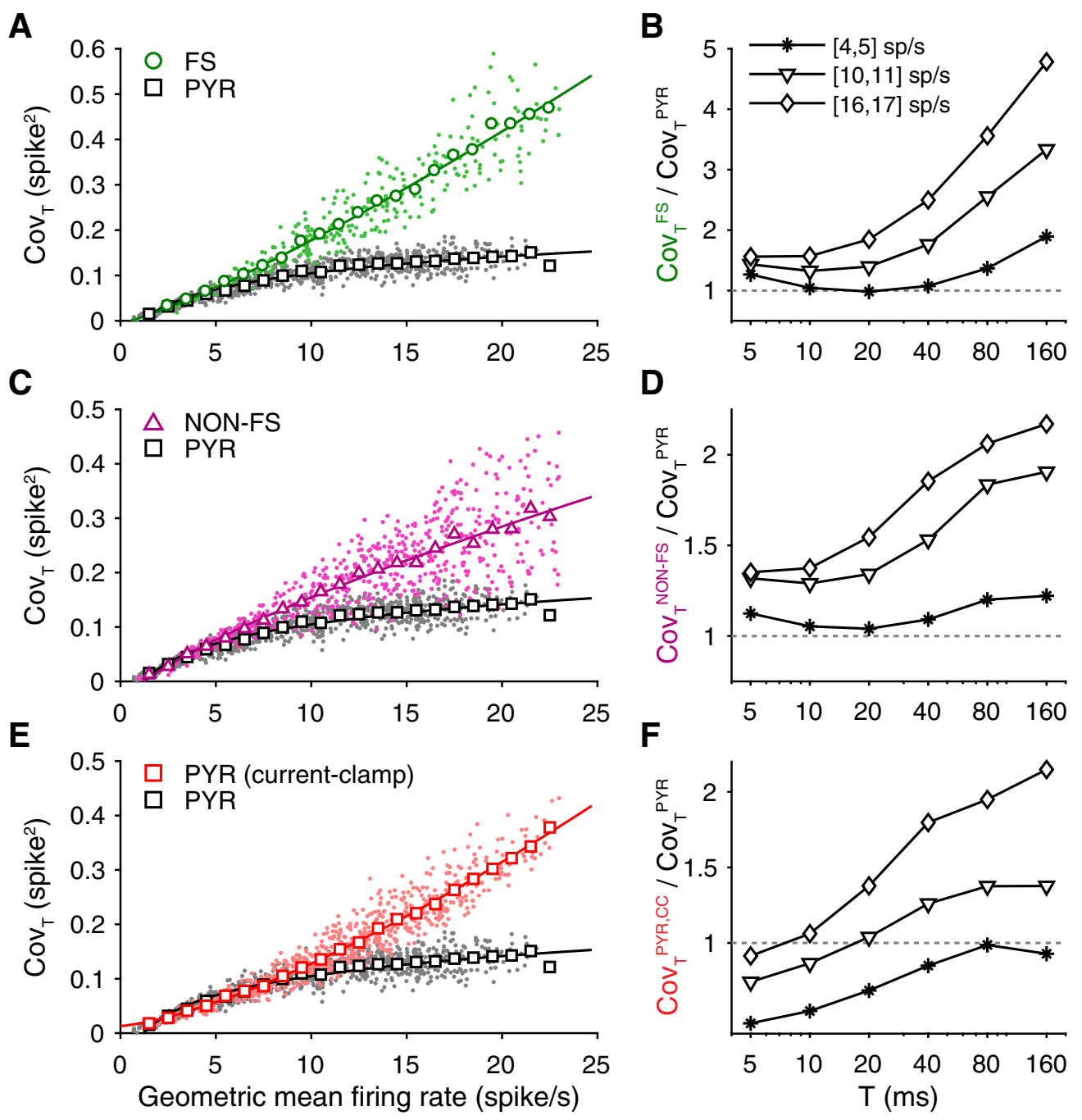

Figure 4. Diversity of correlation transfer across cell type. The covariance of the output spike count was estimated in cell pairs of identical type, for a fixed input correlation $c=0.5$, across distinct firing regimes $(\boldsymbol{A}, \boldsymbol{C}, \boldsymbol{E})$ and spike-count window sizes $(\boldsymbol{B}, \boldsymbol{D}, \boldsymbol{F}) \cdot \boldsymbol{A}$, Each dot is a value of covariance for a given pair of cells and repeated stimulation block, computed over a count window $T=40 \mathrm{~ms}$ and for increasing mean firing rates. Large markers represent the mean of all the individual values, taken in 1 spike/s bins, revealing over our entire dataset a cell-type specific correlation transfer. The solid lines are optimal fits of the binned data with a power-law function of the form $\operatorname{Cov}(f)=a \cdot f^{b}+c$, where $f$ is the geometric mean firing rate of the pair and $a, b$ and $c$ are free parameters. Gray dots and black markers and lines refer to pyramidal cells $(N=26$, with a total number of 855 pairs), while light green dots and green markers and lines refer to $F S$ interneurons $(N=$ 14 , with 285 total number of pairs). $\boldsymbol{B}$, Quantification of covariance shaping in pyramidal cells and FS interneurons, across different spike counting window sizes, for firing frequency regimes in the ranges $[4,5],[10,11]$ and $[16,17]$ spike/s: note how FS interneurons transfer up to 4 times more input correlations than pyramidal cells, over long time-scales and for similar firing rates. $\boldsymbol{C},-\boldsymbol{D}$, Same as $\boldsymbol{A}$ and $\boldsymbol{B}$, but comparing pyramidal cells and NON-FS interneurons, shown with light purple dots and purple markers and lines ( $N=15$, with a total number of 621 pairs). $\boldsymbol{E}$, $\boldsymbol{F}$, Same as $\boldsymbol{A}$ and $\boldsymbol{B}$, but comparing pyramidal cells subjected to conductance-clamp (same data as in $\boldsymbol{A}$ and $\boldsymbol{B}$ ) and current-clamp stimulation, shown with light red dots and red markers and lines $(N=11$, with a total number of 716 pairs). Figures 5 and 6 report representative membrane potential traces, the coefficient of variation of the ISI distributions, respectively. Figure 7 repeats the analysis for mixed cell pairs.

large apical trunk emanating from the cell body and oriented toward the pial surface. From an electrophysiological point of view, pyramidal neurons constituted a homogeneous group, characterized by a regular spiking phenotype with a variable degree of spike-frequency adaptation (accommodation index $0.0083 \pm 0.002$, see Fig. $1 A, D$ ).

Interneurons were morphologically characterized by the absence of visible dendritic processes emanating from the cell body and presented either rounded or elongated shapes. Based on their distinct electrophysiological properties, they could be subdivided into two non-overlapping classes. The main features of the first interneuron type ( $n=23$; see Fig. $1 B)$ were the short AP duration measured at half-width $(0.38 \pm 0.01 \mathrm{~ms})$, the high maximal firing rate achievable upon current injection, typically in excess of 200 $\mathrm{Hz}$, the almost complete lack of spike-frequency adaptation (ac- commodation index $0.0014 \pm 0.0003$; see Fig. $1 B, E)$ and the high values of sag ratio $(0.94 \pm 0.005)$ indicating very little sag in response to hyperpolarizing current pulses. Together, these features identify this neuronal type as a fast-spiking (FS) interneuron (Kawaguchi and Kondo, 2002; Markram et al., 2004; Petilla Interneuron Nomenclature et al., 2008; Tateno and Robinson, 2009; Sippy and Yuste, 2013), whose most abundant representative in the cortex is the parvalbumin-positive basket cell (Wang et al., 2002).

The second interneuron type $(n=29$; see Fig. $1 C)$ was characterized by slightly broader APs $(0.53 \pm 0.02 \mathrm{~ms})$, sag ratios comparable to those of pyramidal cells $(0.77 \pm 0.02)$, strong spike-frequency adaptation (accommodation index $0.02 \pm 0.005$; see Fig. $1 C, F$ ), and rebound spikes in response to hyperpolarizing current steps, present in 20 out of 30 cells in this class (see Fig. 2D). Together, these intrinsic properties suggest 
that this cell type can be identified as a non-fast-spiking (NON-FS) interneuron (Goldberg et al., 2004; Ma et al., 2006; Petilla Interneuron Nomenclature et al., 2008), such as somatostatin-positive Martinotti cells (Kawaguchi and Kondo, 2002; Wang et al., 2002; Silberberg and Markram, 2007; Sippy and Yuste, 2013).

Figures 1 and 2 summarize the main electrophysiological features of the three cell types considered in this study. As mentioned in the Materials and Methods section, for a subset of cells (13 pyramidal, $10 \mathrm{FS}$ and 14 NON-FS) we measured the stationary frequency-current $(F-I)$ curve, with and without the additional injection of background synaptic activity recreated by dynamic-clamp. FS cells had the steepest $F-I$ curves, followed by NON-FS and by pyramidal cells (see Fig. $1 G-I$ ). This ordering was observed both in the "noisefree" condition (empty markers in Fig. $1 G-I)$ - i.e., when only DC current steps were injected into the cell-and in the condition in which the DC steps were applied on top of recreated synaptic background (solid markers in Fig. $1 G-I$ ). These results are consistent with the divisive effect on the slope of the $F-I$ curves produced by a balanced background synaptic activity (Chance et al., 2002). Another effect attributable to the dynamic-clamp stimulation is the reduction in the measured membrane time constant. This amounted to: for pyramidal cells, $22.2 \pm 0.6 \mathrm{~ms}$ to $9.8 \pm 1 \mathrm{~ms}, \mathrm{p}<$ $10^{-10}$, for FS interneurons $6.1 \pm 0.4 \mathrm{~ms}$ to $1.4 \pm 0.1 \mathrm{~ms}, \mathrm{p}<$ $10^{-10}$ and for NON-FS interneurons $17.7 \pm 1.6 m s$ to $3.9 \pm 0.3$ $m s, p<10^{-10}$, mean \pm SEM, two-sample Kolmogorov-Smirnov test.

\section{Output correlation depends on the fraction of common inputs}

We first investigated the dependence of output spike-count correlation $\rho_{T}$ ( $T$ is the window length over which spikes are counted) on the degree of input correlation $c$, in the three cell types previously described. To this end, we performed conductance-clamp experiments and varied $c$ in the range $[0,1]$ (see Fig. $3 A-C$ ), while measuring spike-count correlations using a time window of length $T=40 \mathrm{~ms}$ : we chose this specific value of $T$ because it constitutes a good compromise between the detection of firing rate synchronicity (low values of $T$ ) and covariability (high values of $T$ ). In this set of experiments, we kept the firing rate of the cells constant, in the range $[10,12] \mathrm{Hz}$, to minimize the effects attributable to the dependency of $\rho_{T}$ on the cells' firing rate (de la Rocha et al., 2007) and to facilitate the comparison across cell types. The results of this first set of experiments are shown in Figure 3: similarly to what was described previously for pyramidal cells under a current-clamp stimulation (de la Rocha et al., 2007), $\boldsymbol{\rho}_{T}$ increases monotonically with $c$ under conductance-clamp stimulation, while always remaining smaller than the input correlation value (see Fig. $3 A$ ).

Additionally, for values of input correlation lower than $0.5, \rho_{T}$ depends approximately linearly on $c$ for all three cell types (see Fig. $3 B-D$ ), albeit the linearity is more marked in pyramidal cells, where it extends up to $c=0.5$. These results indicate that, in terms of input-output transfer of correlations, the three neuronal types behave similarly and that, given the linearity of $\rho_{T}$ for low values of input correlation $c$, the linear response theory previously developed (de la Rocha et al., 2007; Litwin-Kumar et al., 2011) and summarized in the Materials and Methods section is suited for interpreting the experimental data.

\section{Spike-count covariance depends on cell pairs type}

By definition, evaluating the spike-count correlation involves computing the covariance between the spike counts from neuron pairs and then normalizing by their respective variances. Linear response theory is only used to approximate the covariance (in the regime where it is small relative to the variances). By contrast, building a theory for the output variance of a neuron where complex cellular processes, such as spike frequency adaptation, that persist beyond a single spike is often difficult (Richardson, 2009; Naud and Gerstner, 2012; Deger et al., 2014; Ocker and Doiron, 2014). For this reason, we now focus on experimentally measuring and understanding the spike-count covariance between a neuron pair.

We investigated whether pyramidal cells and FS and NON-FS interneurons behave similarly in terms of covariance transfer, over a range of geometric mean firing rates. To this end, we fixed $c=0.5$ and varied the ratio of excitatory to inhibitory presynaptic firing rates (Eq. 5) to span (geometric) mean firing rates of the pairs of cells over the range $[0,25] \mathrm{Hz}$. For sufficiently irregular spike trains the absolute magnitude of spike-count covariation increases with the length of the window $T$. It is then convenient to examine the values of spike-count covariance for a given value of $T$ while comparing two conditions, e.g., different cell types or distinct stimulation paradigms.

We first found that cortical interneurons display spike-count covariance values that are higher than pyramidal cells when using a conductance clamp stimulation, over a broad range of firing rates (see Fig. $4 A, C$ ) and for $T$ in the range 5 to $160 \mathrm{~ms}$ (see Fig. $4 B, D)$. In particular, the ratio between interneuron and pyramidal covariances is larger at higher values of geometric mean firing rates (compare diamond-shaped with asterisk markers in Fig. 
A

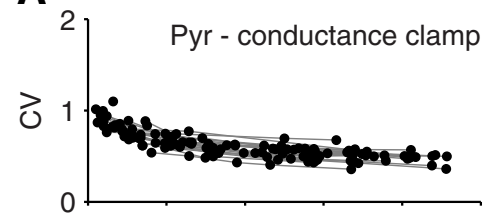

C

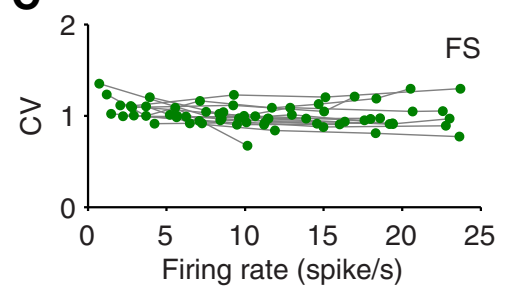

B

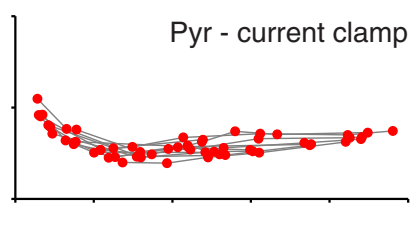

D

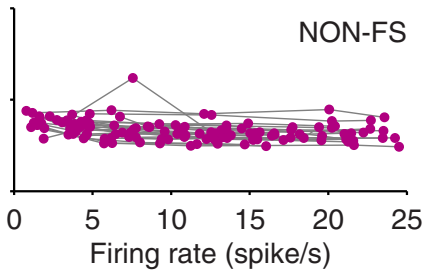

Figure 6. Coefficient of variation (CV) of the ISIs across cell types, upon conductance and current stimulation. For each stimulation block used to compute the values of covariance shown in Figure 4, we extracted the average CV of the ISIs and plotted it against the mean firing rate of the cell in that specific stimulation block. $A, C V$ as a function of mean firing rate for pyramidal cells upon conductance clamp stimulation. $\boldsymbol{B}, \mathrm{CV}$ as a function of mean firing rate for pyramidal cells upon current clamp stimulation. $\boldsymbol{C}$, Same as $\boldsymbol{A}$ but for FS interneurons. $\boldsymbol{D}$, Same as $\boldsymbol{A}$ but for low-threshold-spiking NON-FS interneurons.

$4 B, D)$, and it increases with the value of $T$, reaching values of approximately 5 and of 2 times the covariance of pyramidal cells for FS and NON-FS interneurons, respectively. Interestingly, even when considering mixed cell-type pairs (e.g., one pyramidal cell and one FS interneuron, as shown in Fig. $7 A, B$ ), the resulting covariance values are always higher than what we observed in pyramidal pairs (see Fig. 7).

Together, these results indicate that both interneuron types have a greater capability of transmitting covariation than pyramidal cells, especially on longer timescales and for higher geometric mean firing rates.

\section{Spike-count covariance in pyramidal cells depends on the stimulation paradigm}

In a second set of experiments, we quantified the extent to which a conductance-clamp stimulation shapes the transfer of covariance, compared with a more conventional current-clamp stimulus. To this end, we performed additional recordings in pyramidal cells, employing the current-clamp stimulation described in the Materials and Methods, matching the protocol used by de la Rocha et al. (2007).

We found that for the specific window used $(T=40 \mathrm{~ms})$, covariance transfer in conductance clamp is significantly lower than in current-clamp, at geometric mean firing rates above approximately $10 \mathrm{spike/s}$ (see Fig. $4 E$ ). When performing the same analysis for different values of $T$, the phenomenon of covariance shaping became particularly prominent (see Fig. $4 F$ ). In particular, for short time windows $T$ the spike-count covariance was higher when employing conductance-clamp stimulation, whereas on longer timescales it was higher in current clamp, especially at higher geometric firing rates.

\section{Single-cell properties predict cell-type-specific correlation transfer}

When input correlations $c$ are sufficiently small there is an approximate linear relation between spike correlation $\rho_{T}$ and $c$ (see Fig. 3). Previous work has leveraged this observation to give a simple theory that expresses the spike-count covariance between two neurons $\operatorname{Cov}\left(n_{1}^{T}, n_{2}^{T}\right.$ in terms of single-cell transfer properties (de la Rocha et al., 2007; Litwin-Kumar et al., 2011) (see Materials and Methods). For large values of $T$, the window over which the spike counts $n_{1}^{T}, n_{2}^{T}$ are estimated, this theory reduces to:

$$
\lim _{T \rightarrow \infty} \frac{\operatorname{Cov}\left(n_{1}^{T}, n_{2}^{T}\right)}{T} \approx c \sigma^{2} \cdot \text { gain }_{1} \cdot \text { gain }_{2}
$$

where gain and gain $_{2}$ are the slopes of the frequency-current curves of the two neurons. Indicating by $\mu_{1}$ and $\mu_{2}$ the mean amplitudes of the current inputs experienced by the two neurons, this is formally written as gain ${ }_{i}=d r_{i} / d \mu_{i}$ for $i=\{1,2\}$. Then, the functions $n_{i}^{T}=\operatorname{Tr}_{i}\left(I_{i}^{T}\right)$ link the integrated input experienced by each neuron $I_{i}^{T}=\int_{0}^{T} I_{i}(t) d t$ to its output spike count. Since $\mu_{i}=\lim _{T \rightarrow \infty} I_{i}^{T} / T$ and $r_{i}$ $=\lim _{T \rightarrow \infty} n_{i}^{T} / T$ then, for large $T, r_{i}\left(\mu_{i}\right)$ is the frequency-current curve that we measured (see Fig. $1 G-I$ ), and $d r_{i} / d \mu_{i}$ is the firing rate gain. In other words, Eq. 1 approximates $\operatorname{Cov}\left(n_{1}^{T}, n_{2}^{T}\right)$ as the input covariance $c \sigma_{2}$ scaled by the product of firing rate gains.

If $r_{i}\left(\mu_{i}\right)$ is linear, then the gain $d r_{i} / d \mu_{i}$ remains fixed as $\mu_{i}$ changes, so that $\operatorname{Cov}\left(n_{i}^{T}, n_{j}^{T}\right)$ also does not shift. By contrast, a nonlinear $r_{i}\left(\mu_{i}\right)$ makes $\operatorname{Cov}\left(n_{i}^{T}, n_{j}^{T}\right)$ depend on the "operating point" of the neuron pair (measured by $d r_{i} / d \mu_{i}$ ), so that $\operatorname{Cov}\left(n_{i}^{T}, n_{j}^{T}\right)$ is malleable despite the input covariance remaining fixed at $\mathrm{Co}_{2}$. Intuition for this effect comes from an understanding of how the joint input density $p\left(I_{1}^{T}, I_{2}^{T}\right)$ is mapped to the joint output density $p\left(n_{1}^{T}, n_{2}^{T}\right)$. The Gaussian density of $p\left(I_{1}^{T}, I_{2}^{T}\right)$ is reshaped by the nonlinear mapping $r_{1}\left(I_{1}^{T}\right)$ and $r_{2}\left(I_{2}^{T}\right)$ of each cell in the pair, so that the density $p\left(n_{1}^{T}, n_{2}^{T}\right)$ is non-Gaussian (see Fig. $8 A)$. Nevertheless, $\operatorname{Cov}\left(n_{1}^{T}, n_{2}^{T}\right)$ reports the effective spread of $p\left(n_{1}^{T}, n_{2}^{T}\right)$. along the diagonal $n_{1}=n_{2}$. For low $\mu_{1}$ the spike threshold nonlinearity forces both the output firing rate $n_{1} / T$ and the gain $d r_{i} / d \mu_{i}$ to be small, compromising the spread of $p\left(n_{1}^{T}, n_{2}^{T}\right)$. When $\mu_{1}$ is increased, the transfer of common fluctuations is enhanced through sampling larger firing rate gains in the $n_{1}=\operatorname{Tr}\left(I_{1}^{T}\right)$ transfer. This results in an increased spread of $p\left(n_{1}^{T}, n_{2}^{T}\right)$ along $n_{1}=n_{2}$, and naturally $\operatorname{Cov}\left(n_{1}^{T}, n_{2}^{T}\right)$ is larger. Thus, by controlling the gain of the firing rate transfer (through $\mu_{1}$ and $\left.\mu_{2}\right)$ the output $\operatorname{Cov}\left(n_{1}^{T}, n_{2}^{T}\right)$ is manipulated despite the input $\operatorname{Cov}\left(I_{1}^{T}, I_{2}^{T}\right)$ being fixed.

We next used this theory to investigate how the single-cell properties of pyramidal, FS, and NON-Fcell types determine $\operatorname{Cov}\left(n_{1}^{T}, n_{2}^{T}\right)$, and how it changes with firing rate. Exponential integrate-and-fire models with slow adaptation currents (Badel et al., 2008; Brette et al., 2008) were matched to the passive and firing rate characteristics of the experimentally measured pyramidal, FS, and NON-FS cells (see Fig. 8B) (see Materials and Methods for details). Mimicking the experiments, we employed a stochastic bombardment of presynaptic excitatory and inhibitory conductance inputs to drive stochastic spike train responses (see Fig. $8 B$ ). The simplicity of the spike generation mechanism combined with vanishing synaptic timescales and large pre-synaptic ensembles permits a theoretical calculation of the firing rate curves $r_{i}\left(\mu_{i}\right)$ and their gains $d r_{i} / d \mu_{i}$ (see Materials and Methods). We first computed the $F-I$ curves by injecting static currents while fixing the input variance (see Fig. $4 C$ ), qualitatively matching the experimental results (cf. Figs. $8 C, 1 G-I$ ). The FS cells, 
however, had more similar F-I curves to the NON-FS and pyramidal cells in our model than in the averaged experimental $F-I$ curves, perhaps due to the different noise levels (see Materials and Methods).

To probe the input-output transfer function, we next increased the output firing rates $r_{i}$ by varying the presynaptic excitatory input rate, $R_{E}$. For the fitted cellular and synaptic parameters the model firing rate curves qualitatively matched those reported for pyramidal, FS, and NON-FS cells. For all models the firing rate gain $d r_{i} / d \mu_{i}$ increased with the output firing rate of the cells, with FS interneurons showing the highest dependence (see Fig. $8 D$ ). Furthermore, as output firing rates were driven by a stochastic bombardment of presynaptic inputs, then as the output firing rate increased so did the overall input variance $\sigma_{2}$, again for all models (see Fig. 8E).

Finally, we modeled a pair of cells receiving correlated conductance fluctuations ( $c=0.1$ and numerically estimated $\operatorname{Cov}\left(n_{1}^{T}, n_{2}^{T}\right)$ as firing rates varied. Our simulations recapitulated the experimental results, namely at high firing rates FS interneuron pairs showed the largest covariance, followed by NON-FS interneuron pairs, and with pyramidal cell pairs having the lowest covariance (see Fig. $8 F$, open circles). Our theory based on single-cell properties also gave a quantitative match to $\operatorname{Cov}\left(n_{1}^{T}, n_{2}^{T}\right)$ for all cell types (see Fig. $8 F$, solid lines). This provides evidence that the cell-type differences in the electrical properties that determine single-cell firing rate gain (see Fig. $8 D$ ) underlie, in part, the cell-type distinctions in covariance transfer (see Fig. $8 F$ ).

Interestingly, the pyramidal cell model described previously qualitatively captures the shaping of the covariance values observed in the experiments (Fig. 8G,H). However, how correlation shaping depends on the cell pair firing rates is reversed in the theory compared with the experiments. More to the point, in the experiments the highest values of $\left.\operatorname{Cov}_{T}^{\text {pyr,cc }} / \operatorname{Cov}_{T}^{\text {pyrgc }}\right)$ are observed for high values of geometric mean rate (in the range [16,17] $\mathrm{Hz}$, diamond markers in Fig. $4 F$ ) and gradually decrease for decreasing geometric mean rates. In theory and in simulations, on the other hand, high geometric mean firing rates correspond to lower values of covariation shaping (diamond markers in Fig. $8 H$ ). In both theory and experiment the raw $\operatorname{Cov}\left(n_{1}^{T}, n_{2}^{T}\right)$ values increase with firing rates (see Figs. $4 E, 8 G$ ), yet in the theory there is a saturation in $\operatorname{Cov}\left(n_{1}^{T}, n_{2}^{T}\right)$ with firing rates in current clamp that is absent in the in vitro recordings (and vice versa for the conductance-clamp case). These discrepancies may be attributed to cellular processes that are ignored in our model, such as subthreshold voltage-gate conductances whose activation differs between current- and conductance-clamp activation in real neurons.

\section{Measured covariance values correlate with single- cell properties}

Our linear response theory predicts that the magnitude of covariance between two spike trains from distinct neurons should depend on the slopes of their $F-I$ curves (Eq. 23). If the F-I curves
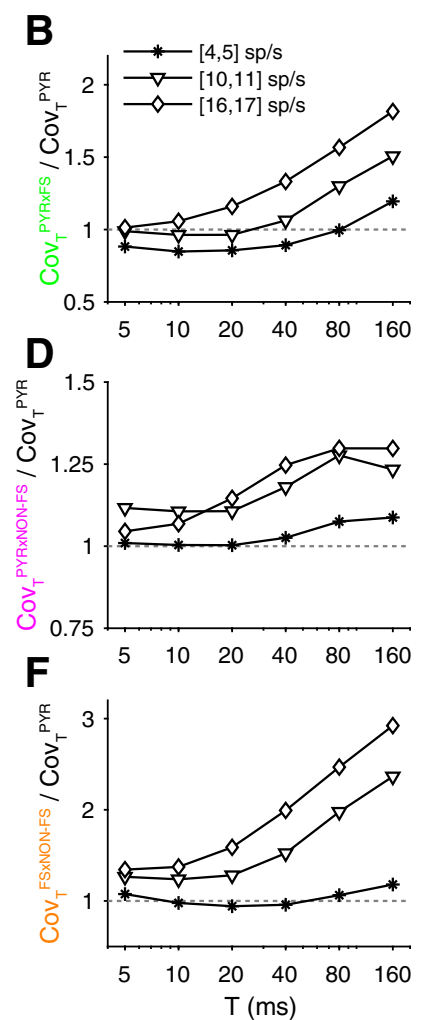

ce values computed in heterogeneous pairs of cells: pyramidal and FS interneurons ( $\boldsymbol{A}$, green markers and traces), pyramidal and NON-FS interneurons ( $\boldsymbol{C}$, purple markers and traces) and FS and omogenous pairs composed of two pyramidal cells (black markers and traces in all panels, same data as in Fig. 4). B, D, $\boldsymbol{F}$ Covariance shaping of mixed pairs of cells. Same analysis as that of Figure 4.

were truly linear then the spike train covariance would be a fixed value, independent of the firing rates of the neuron pair. However, in fluctuation driven regimes the $F-I$ curves are markedly nonlinear, with a slope that grows with the firing rate (see Fig. $9 A-C)$. Thus, by changing the firing rates of a neuron pair we sample a range of gains and associated pairwise covariances by varying the cell pair firing rates. This in turn provides an opportunity to quantitatively test our linear response prediction: for each pair of cells in our dataset we computed the coefficient of determination $r^{2}$ between the measured covariance values and the product of the slopes of the $F-I$ curves of the individual cells, computed with the injection of recreated background synaptic activity, as shown in Figure 1, $G$ to $I$, at the population level (filled markers) and in Figure 9, $A$ to $C$, for the representative cells used in Figure 9, $D$ to $F$. In agreement with the prediction of the linear response theory, we found a very strong correlation between the covariance and the product of the $F-I$ curve gains, on a cell pairby-cell pair basis (see Figs. 9D-F, 10A-C), and for each of the three cell types under analysis. Interestingly, the distribution of coefficients $r^{2}$ (see Figs. 9G-I, Fig. 10D-F) was more strongly skewed toward high values for the FS interneuron cell type, indicating that the covariation dynamics of GABAergic fast-spiking interneurons are better captured by our linear response theory, at least for the intermediate value of input correlation used in these experiments (i.e., $c=0.5$ ). Together, these results demonstrate how microcircuit observables - the magnitude of covariation in the spike trains of two cells — can be related to intrinsic, single cell properties of the neurons that constitute the microcircuit-in this case, the slope of their F-I curves. 
A

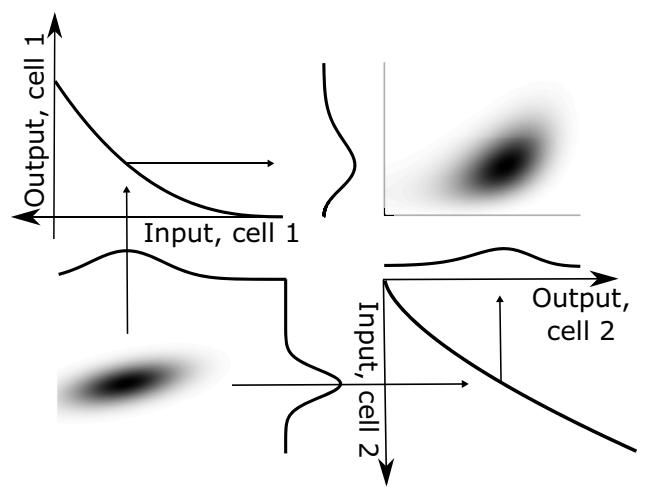

C

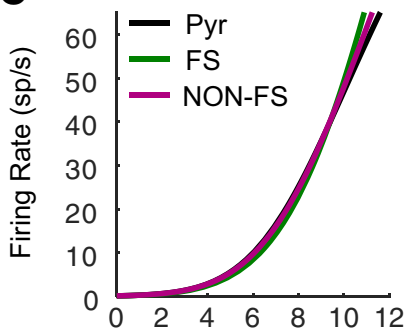

Current from rheobase $\left(\mu \mathrm{A} / \mathrm{cm}^{2}\right)$

F

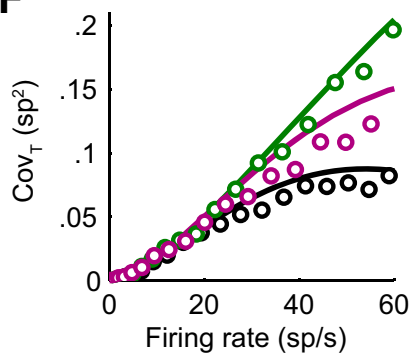

B
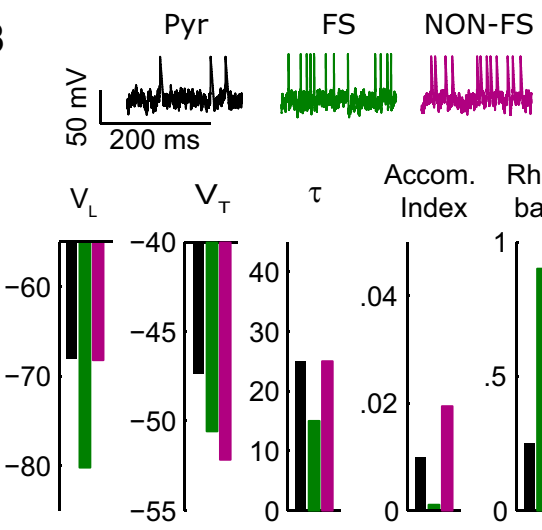

Accom. Rheo-

Index base

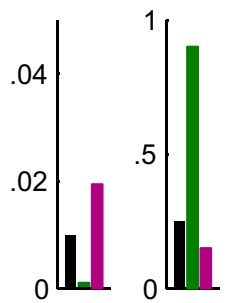

D

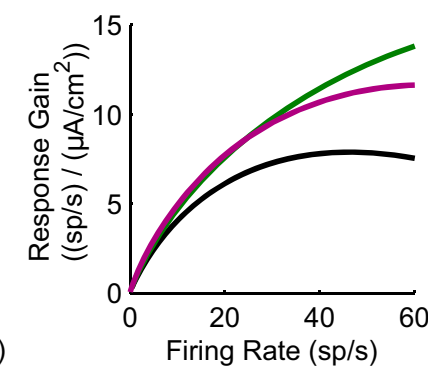

E
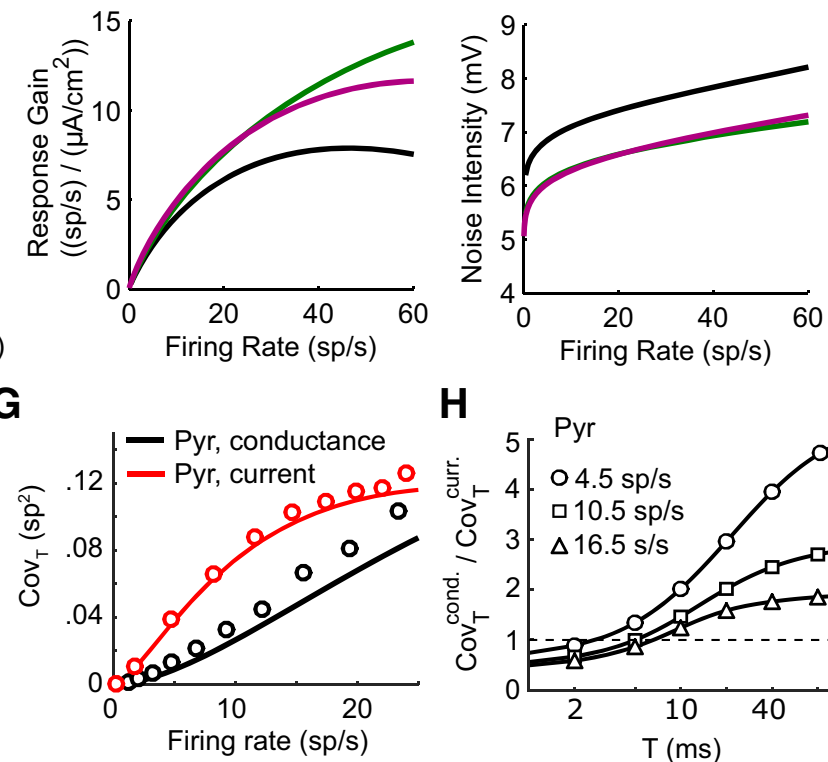

H

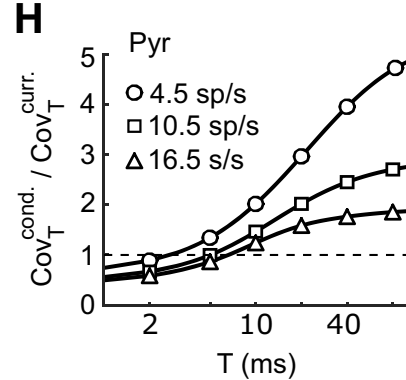

Figure 8. Single-cell properties predict covariance transfer. A, Schematics of covariance transfer with two cells. A correlated Gaussian input (bottom left) is transferred through each cell's $F-I$ curve (top left, bottom right) to produce a correlated, non-Gaussian output (top right). Linear response theory predicts the covariance of that output distribution by linearizing the $F-I$ curves around the stimulus mean. $\boldsymbol{B}$, Cell-type-specific adapting exponential integrate-and-fire models match the recorded single-cell properties. Units: Leak reversal potential $\left(V_{L}\right)$ and spike threshold $\left(V_{T}\right)$ in $\mathrm{mV}$; passive membrane time constant $(\tau)$ in $\mathrm{ms}$; rheobase in nA. C, Static transfer functions ( $F-I$ curves) for each model. $D$, Slope of the $F-I$ curve as a function of firing rate when the rate of the presynaptic excitatory population is increased (see Materials and Methods: "Neuron models"). $\boldsymbol{E}$, Intensity of the input noise as the rate of the presynaptic excitatory population is increased. $\boldsymbol{F}$, Spike count covariance versus geometric mean output rate for each cell type. $\boldsymbol{G}$, Spike count covariance versus firing rate. $\boldsymbol{H}$, Comparison of pyramidal model cells subjected to conductance-clamp or current-clamp stimuli (as in Figure 4F).

\section{Discussion}

We have shown that three distinct cell types-pyramidal cells and two types of GABAergic interneurons-in L5 of rat somatosensory cortex respond differently to the same level of correlated inputs. In our experiments, cells were stimulated with biophysically realistic conductance inputs that mimic the activation of synaptic receptors and that recapitulate the features of the highconductance state observed in vivo (Destexhe et al., 2001). It is worth pointing out that our results could not have been obtained using current-clamp alone, as they crucially depend on the modulation of intrinsic cell properties that can only be ascribed to conductance injection. To the best of our knowledge, this is the first time that differences have been found in the capability of distinct neuronal types to transmit input correlations in a firing rate-dependent manner.

\section{Cell-type-specific differences in covariation transfer}

We interpreted our experimental data through a theory that predicts how spiking neurons transfer co-fluctuations in inputs to their output spike trains (Litwin-Kumar et al., 2011). We observed that pairs of FS interneurons display a degree of covariation that is several factors larger than pairs of pyramidal cells, while pairs of NON-FS interneurons produced covariation values that were only slightly larger than pyramidal cells. Interestingly, this can be explained by an intrinsic cellular property-the greater steepness of FS interneurons' static input-output relationship than pyramidal cells-therefore tying a single-cell feature to a network observable. Although our experiments were carried out in juvenile rats, which may not have reached yet a complete degree of neuronal maturation, the relative ordering of the steepness of the $F-I$ curves of the three cell types considered here is preserved also in adulthood (Jiang et al., 2015), thus making our main results valid beyond the juvenile stage investigated in the present study.

Although our work highlights the importance of cellular differences between cell classes for correlation transfer, it ignores the specifics of how pyramidal cells and FS and NON-FS interneurons are positioned within the recurrent cortical circuit (Pfeffer et 

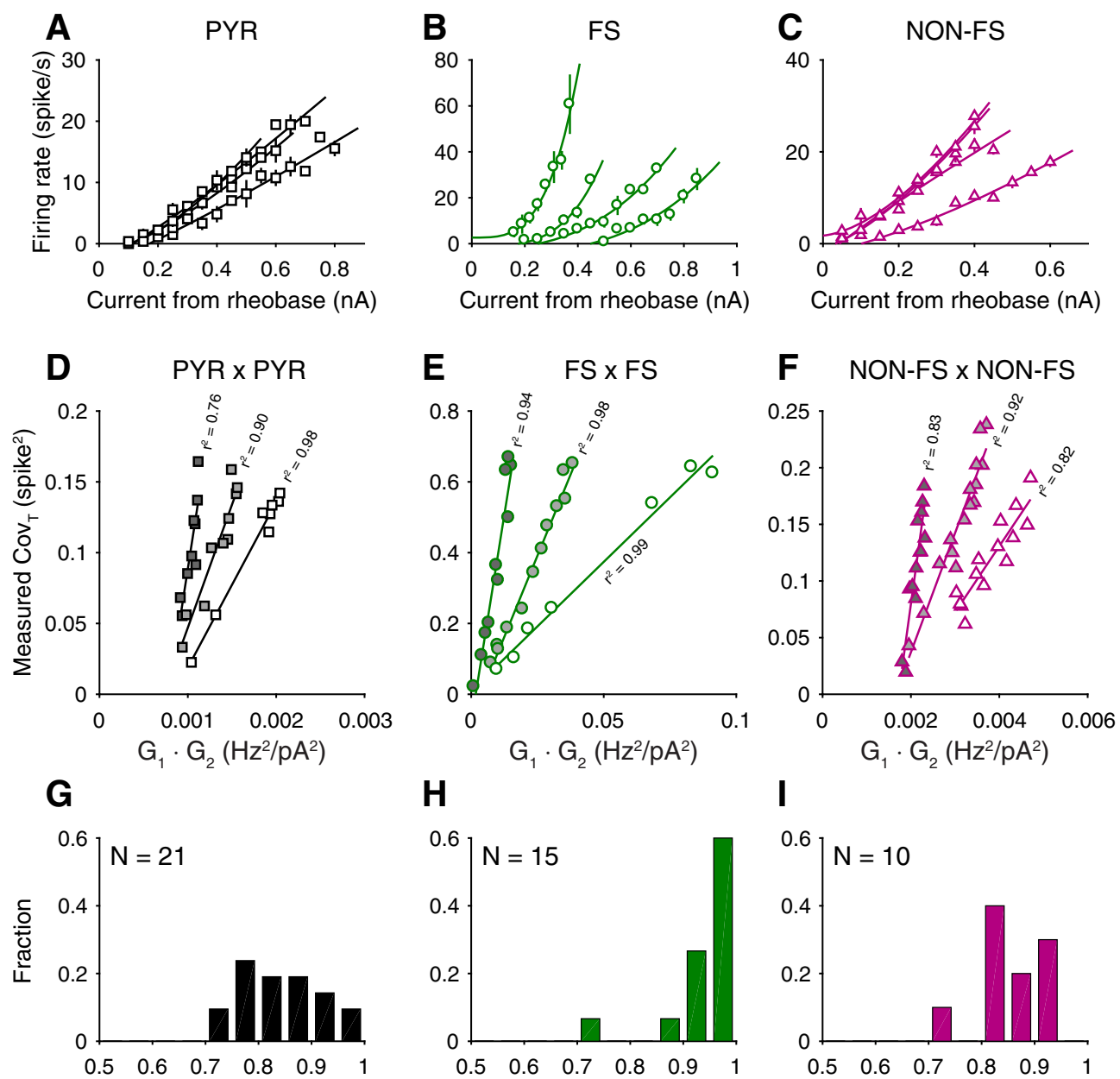

Coefficient of determination $r^{2}$ Coefficient of determination $r^{2}$ Coefficient of determination $r^{2}$

Figure 9. The product of the slopes of the $F-I$ curves strongly correlates with the measured covariance values, on a cell-pair by cell-pair basis and in a frequency-dependent manner For each cell pair, we computed several covariance values corresponding to different geometric mean firing rates. We plotted them as a function of the product of the slopes of the $F-I$ curves of each cell of the pair (i.e., gain ${ }_{1}$ and gain ${ }_{2}$ ), at the corresponding value of firing rate. This uncovers a strong correlation between measured covariance and product of gain ${ }_{1}$ and gain $_{2}$, as predicted by the linear response theory. $\boldsymbol{A}$, Individual $F-I$ curves upon injection of constant steps of current in addition to recreated background synaptic activity for the pyramidal cells shown in $\boldsymbol{D}$. $\boldsymbol{B}, \boldsymbol{C}$, Same as $\boldsymbol{A}$, but for the FS and NON-FS cells whose covariance values versus product of $F-I$ gains are shown in $\boldsymbol{E}$ and $\boldsymbol{F}$, respectively. $\boldsymbol{D}$, Representative examples of correlation between measured covariance and slopes of the $F-I$ curves, for three pairs of pyramidal cells. Actual values are shown with square markers, while solid lines are linear fits to the data. The $r^{2}$ coefficients are indicated in the panel for each cell pair. $\boldsymbol{E}, \boldsymbol{F}$, Same as $\boldsymbol{D}$, but for FS and NON-FS interneurons, respectively. $\boldsymbol{G}$, Distribution of $r^{2}$ coefficients for $N=20$ pairs obtained from eight pyramidal cells. $\boldsymbol{H}, I$, Same as $\boldsymbol{G}$ but for $F S(N=15$ pairs from 6 cells) and NON-FS interneurons ( $N=10$ pairs from 5 cells), respectively. The value of the spike counting window is $T=40 \mathrm{~ms}$ in all panels. Figure 10 repeats this analysis for mixed-cell pairs.

al., 2013; Tremblay et al., 2016). Linear response theory has a natural extension to recurrently coupled networks of spiking neurons (Ocker et al., 2017), where single neuron transfer functions are mixed with wiring structure to determine network correlations. However, previous studies that employ this theory have ignored differences in inhibitory neurons and rather simply model a single interneuron class (Bos et al., 2016). Furthermore, most modeling studies that include multiple interneuron subtypes have only focused on firing rate models (Litwin-Kumar et al., 2016; Garcia Del Molino et al., 2017; Kuchibhotla et al., 2017), and they do not discuss how fluctuations are distributed over a network. The cellular insights exposed in our study when combined with established recurrent circuit theory promise new insights into how biologically realistic cortical circuits produce and transfer network-wide correlations.

\section{Role of conductance-clamp stimulation}

Our conductance-clamp stimulation protocol was instrumental in clarifying how the modulation of spike-count covariance by the geometric mean firing rate strongly depends on the cell type. This is in contrast with what was shown in earlier experiments using a current-clamp stimulus alone (de la Rocha et al., 2007), where the authors found no major differences among regular spiking and intrinsic bursting pyramidal cells and fast-spiking interneurons.

To understand this discrepancy, we explicitly compared conductance and current-clamp stimulations in pyramidal cells (see Fig. $4 E-F$ ). Our results are in agreement with the correlation-shaping mechanism first presented in (LitwinKumar et al., 2011), namely that high conductance states better transfers short timescale covariability, while a low conductance state (comparable to current clamp) better transfers long timescale covariability. However, in that study in vitro neurons were held at a constant firing rate, whereas here we explored a range of firing frequencies. Our work highlights that for higher geometric mean firing rates the increased covariability transfer at short timescales in the high conductance state is diminished. This should be contrasted with the in- 

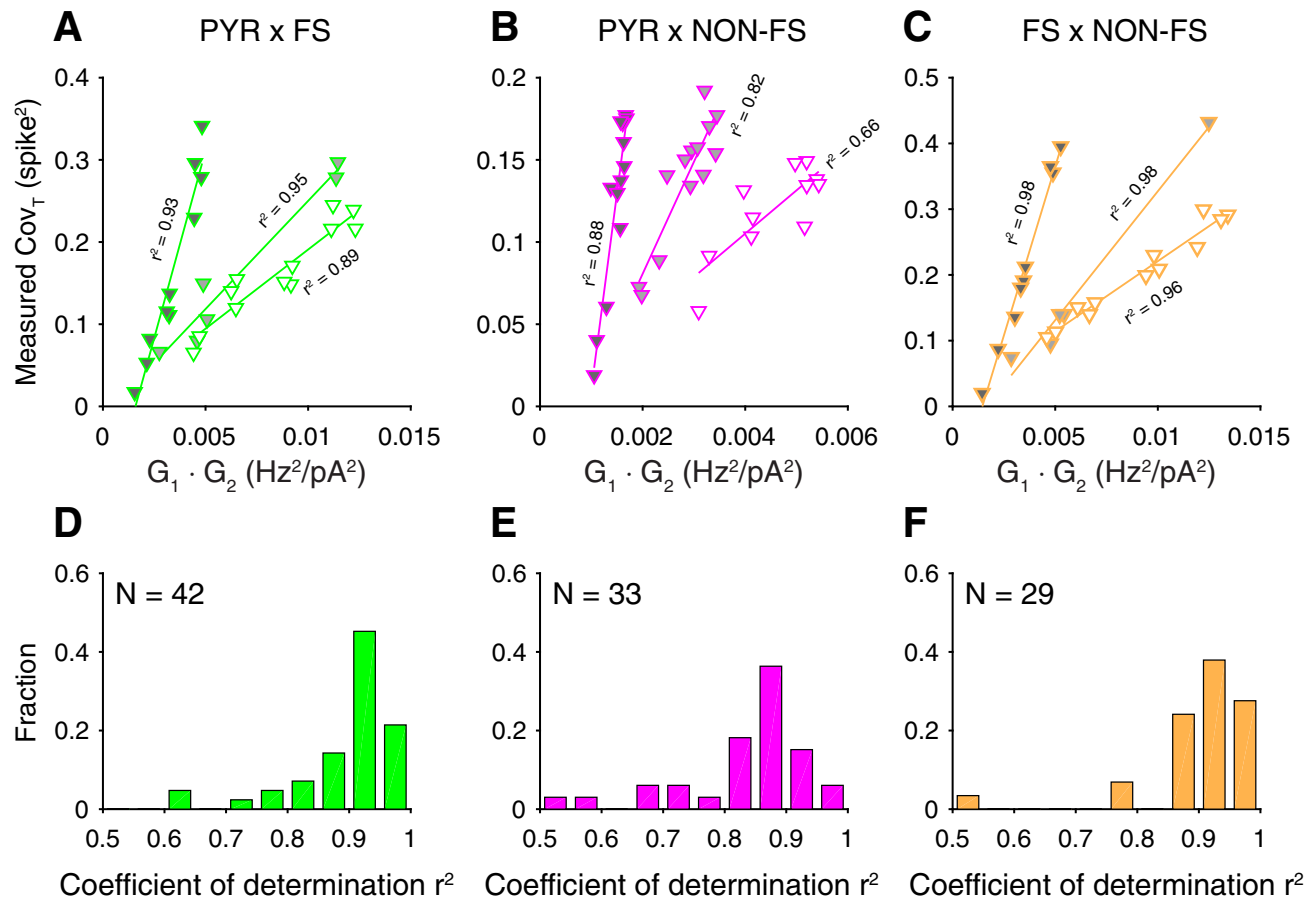

Figure 10. Correlation between the product of the gain of the $F-I$ curves and the measured covariance values, in mixed cell pairs. Same analysis as that performed in Figure 8 but for pairs of cells composed of different cell types. Also in this case there is a very strong correlation between the experimentally measured values of covariance and the product of the slopes of the $F-I$ curves of the two cells in the pair. $A$, Representative examples of correlation between measured covariance and slopes of the $F-I$ curves, for three mixed pairs of pyramidal and FS cells. Actual values are shown with square markers, while solid lines are linear fits to the data. The $r^{2}$ coefficients are indicated in the panel for each cell pair. $\boldsymbol{B}, \boldsymbol{C}$, Same as A, but for mixed pairs of pyamidal and NON-FS cells and for mixed pairs of FS and NON-FS interneurons, respectively. D, Distribution of $\mathrm{r}^{2}$ coefficients for the mixed pairs of pyramidal and FS cells. $\boldsymbol{E}, \boldsymbol{F}$, Same as D but for the other mixed pair cases. The Value of the spike counting window is $T=40 \mathrm{~ms}$ in all panels.

creased covariability transfer at long timescales in a low conductance state (current clamp), which is enhanced at high firing rates.

In summary, our experimental and modeling results show that pyramidal neurons in the high-conductance state are more suited to transferring covariation on short timescales (i.e., via synchronous activation) than previously predicted by currentclamp experiments (de la Rocha et al., 2007). This despite the fact that the firing variability, measured as the coefficient of variation of the ISIs, is comparable in these two conditions (see Fig. 6).

Of course, the major limitation of any dynamic-clamp stimulation consists in the point nature of the conductance injection. Although this might be a reasonable assumption for neurons with relatively small dendritic trees, such as basket cells (Wang et al., 2002; Markram et al., 2004), L5 pyramidal cells like the ones considered in this study have extensive dendrites containing a variety of ion channels (Stuart and Sakmann, 1994; Harnett et al., 2015), which, together with nonlinear mechanisms of synaptic integration (Harnett et al., 2012; Xu et al., 2012), support a variety of complex processing tasks (for a review see (Spruston, 2008)). This adds a further dimension to the structure of the inputs, which can be not only temporally correlated, as is the case for the inputs considered in this work, but also spatially correlated, thus leading to potentially interesting phenomena of inputs cooperativity, which largely remain to be explored.

\section{Relating theory and experiment}

De la Rocha and colleagues (de la Rocha et al., 2007) put forth a theory that related the gain of single neuron input-output transfer to how correlated input fluctuations are transferred by neuron pairs to output spike-count correlations (Eq. 23).
However, in that study, as well as subsequent ones, in vitro experiments were only qualitatively compared with theory. For instance, while the dependence of spike-count correlations upon the geometric mean firing rate can be derived from our linear theory at low firing rates (Shea-Brown et al., 2008), it has only been shown to be qualitatively true in real neurons (de la Rocha et al., 2007). Similarly, theoretical work has shown how the timescale (Litwin-Kumar et al., 2011) or excitability class (Barreiro et al., 2010; Hong et al., 2012) of membrane integration determines the timescales over which correlations are transferred, nevertheless experimental tests were only qualitative in nature (e.g., an increase or decrease in correlation as membrane properties are changed).

The difficulty with a quantitative test of a linear response theory of correlations is that a systematic measurement of both neuronal gain and correlations transfer in neuron pairs is rarely performed over a range of firing rates. Indeed, studies that focus upon single neuron gain control explicitly ignore populations of neurons, while studies of neuronal populations often only study the network in a single regime (and hence a single gain value). The results presented in Figure 9 of our study thus constitute the most experimentally validated test of this theory, comparing the mapping between neuronal gain and covariability over a range of gain values. Furthermore, our work uses the heterogeneity over pyramidal cells and FS and NON-FS interneurons to test our theory over a broad range of cellular properties.

\section{Functional implications}

Overall, our results raise the intriguing possibility that principal cells in the neocortex - and possibly in other areas of the brain, such as the hippocampus - might be specifically tuned to operate 
in a low-correlation level, which could improve the coding capabilities of a population of principal cells. Conversely, the higher covariance values measured in the two interneuron classes, and in particular in fast-spiking interneurons, are likely to play a role in the regulation of the activity of local circuits by interneurons. In particular, higher level of correlation might facilitate interneurons in their function as providers of a "blanket of inhibition" (Packer and Yuste, 2011; Karnani et al., 2014), and, in the case of FS interneurons, could be implicated in the important role these cells play in orchestrating cortical oscillations (see (Freund and Katona, 2007) and references therein). Interestingly, it has recently been shown that a class of GABAergic cells in the prefrontal cortex sends long-range projections to subcortical areas (Lee et al., 2014): it would be of great interest to investigate whether these cells present correlation transfer properties that differ from what we have described here.

In conclusion, our findings underline the importance of an in-depth characterization of cortical cell diversity by increased experimental realism and point to a richness of network behaviors arising from the diversity of intrinsic cell properties.

\section{References}

Badel L, Lefort S, Brette R, Petersen CC, Gerstner W, Richardson MJ (2008) Dynamic I-V curves are reliable predictors of naturalistic pyramidalneuron voltage traces. J Neurophysiol 99:656-666.

Bair W, Zohary E, Newsome WT (2001) Correlated firing in macaque visual area MT: time scales and relationship to behavior. J Neurosci 21:16761697.

Barreiro AK, Shea-Brown E, Thilo EL (2010) Time scales of spike-train correlation for neural oscillators with common drive. Phys Rev E Stat Nonlin Soft Matter Phys 81:011916.

Bos H, Diesmann M and Helias M (2016) Identifying anatomical origins of coexisting oscillations in the cortical microcircuit. PLoS Comput Biol 12:e1005132.

Brette R, Gerstner W (2005) Adaptive exponential integrate-and-fire model as an effective description of neuronal activity. J Neurophysiol 94:36373642.

Brette R, Piwkowska Z, Monier C, Rudolph-Lilith M, Fournier J, Levy M, Frégnac Y, Bal T, Destexhe A (2008) High-resolution intracellular recordings using a real-time computational model of the electrode. Neuron 59:379-391.

Brunel N, Chance FS, Fourcaud N, Abbott LF (2001) Effects of synaptic noise and filtering on the frequency response of spiking neurons. Phys Rev Lett 86:2186-2189.

Buzsáki G, Mizuseki K (2014) The log-dynamic brain: how skewed distributions affect network operations. Nat Rev Neurosci 15:264-278.

Chance FS, Abbott LF, Reyes AD (2002) Gain modulation from background synaptic input. Neuron 35:773-782.

Cohen MR, Kohn A (2011) Measuring and interpreting neuronal correlations. Nat Neurosci 14:811-819.

Connors BW, Gutnick MJ (1990) Intrinsic firing patterns of diverse neocortical neurons. Trends Neurosci 13:99-104.

Cox D, Miller H (1965) The theory of stochastic processes. London: Methuen.

Cox DR, Isham V (1980) Point processes. Boca Raton, FL: CRC.

Dayan P, Abbott LF (2001) Theoretical neuroscience. Cambridge, MA: MIT.

de la Rocha J, Doiron B, Shea-Brown E, Josić K, Reyes A (2007) Correlation between neural spike trains increases with firing rate. Nature 448:802806.

deCharms RC, Merzenich MM (1996) Primary cortical representation of sounds by the coordination of action-potential timing. Nature 381:610613.

Deger M, Schwalger T, Naud R, Gerstner W (2014) Fluctuations and information filtering in coupled populations of spiking neurons with adaptation. Phys Rev E Stat Nonlin Soft Matter Phys 90:062704.

Garcia Del Molino LC, Yang GR, Mejias JF, Wang XJ (2017) Paradoxical response reversal of top-down modulation in cortical circuits with three interneuron types. Elife 6:e29742.
Destexhe A, Paré D (1999) Impact of network activity on the integrative properties of neocortical pyramidal neurons in vivo. J Neurophysiol 81:1531-1547.

Destexhe A, Rudolph M, Fellous JM, Sejnowski TJ (2001) Fluctuating synaptic conductances recreate in vivo-like activity in neocortical neurons. Neuroscience 107:13-24.

Destexhe A, Rudolph M, Paré D (2003) The high-conductance state of neocortical neurons in vivo. Nat Rev Neurosci 4:739-751.

Destexhe A and Bal T (2009) Dynamic clamp. New York: Springer.

Doiron B, Litwin-Kumar A, Rosenbaum R, Ocker GK, Josić K (2016) The mechanics of state-dependent neural correlations. Nat Neurosci 19:383-393.

Druckmann S, Banitt Y, Gidon A, Schürmann F, Markram H, Segev I (2007) A novel multiple objective optimization framework for constraining conductance-based neuron models by experimental data. Front Neurosci 1:7-18.

Erisken S, Vaiceliunaite A, Jurjut O, Fiorini M, Katzner S, Busse L (2014) Effects of locomotion extend throughout the mouse early visual system. Curr Biol 24:2899-2907.

Fourcaud N, Brunel N (2002) Dynamics of the firing probability of noisy integrate-and-fire neurons. Neural Comput 14:2057-2110.

Freund TF, Katona I (2007) Perisomatic inhibition. Neuron 56:33-42.

Gentet LJ, Avermann M, Matyas F, Staiger JF, Petersen CC (2010) Membrane potential dynamics of GABAergic neurons in the barrel cortex of behaving mice. Neuron 65:422-435.

Gentet LJ, Kremer Y, Taniguchi H, Huang ZJ, Staiger JF, Petersen CC (2012) Unique functional properties of somatostatin-expressing GABAergic neurons in mouse barrel cortex. Nat Neurosci 15:607-612.

Goldberg JH, Lacefield CO, Yuste R (2004) Global dendritic calcium spikes in mouse layer 5 low threshold spiking interneurones: implications for control of pyramidal cell bursting. J Physiol 558:465-478.

Meyer HS, Wimmer VC, Oberlaender M, de Kock CP, Sakmann B, Helmstaedter M (2010) Number and laminar distribution of neurons in a thalamocortical projection column of rat vibrissal cortex. Cereb Cortex 20:2277-2286.

Harnett MT, Makara JK, Spruston N, Kath WL, Magee JC (2012) Synaptic amplification by dendritic spines enhances input cooperativity. Nature 491:599-602.

Harnett MT, Magee JC, Williams SR (2015) Distribution and function of HCN channels in the apical dendritic tuft of neocortical pyramidal neurons. J Neurosci 35:1024-1037.

Hengen KB, Lambo ME, Van Hooser SD, Katz DB, Turrigiano GG (2013) Firing rate homeostasis in visual cortex of freely behaving rodents. Neuron 80:335-342.

Henze DA, Buzsáki G (2001) Action potential threshold of hippocampal pyramidal cells in vivo is increased by recent spiking activity. Neuroscience 105:121-130.

Higgs MH, Spain WJ (2009) Conditional bursting enhances resonant firing in neocortical layer 2-3 pyramidal neurons. J Neurosci 29:1285-1299.

Holden AV (1976) Models of the Stochastic Activity of Neurones. Models of the Stochastic Activity of Neurones. New York: Springer.

Hong S, Ratté S, Prescott SA, De Schutter E (2012) Single neuron firing properties impact correlation-based population coding. J Neurosci 32: 1413-1428.

Ilin V, Malyshev A, Wolf F, Volgushev M (2013) Fast computations in cortical ensembles require rapid initiation of action potentials. J Neurosci 33:2281-2292.

Jiang X, Shen S, Cadwell CR, Berens P, Sinz F, Ecker AS, Patel S, Tolias AS (2015) Principles of connectivity among morphologically defined cell types in adult neocortex. Science 350:aac9462.

Karnani MM, Agetsuma M, Yuste R (2014) A blanket of inhibition: functional inferences from dense inhibitory connectivity. Curr Opin Neurobiol 26:96-102.

Kawaguchi Y, Kondo S (2002) Parvalbumin, somatostatin and cholecystokinin as chemical markers for specific GABAergic interneuron types in the rat frontal cortex. J Neurocytol 31:277-287.

Kole MH, Stuart GJ (2008) Is action potential threshold lowest in the axon? Nat Neurosci 11:1253-1255.

Köndgen H, Geisler C, Fusi S, Wang XJ, Lüscher HR, Giugliano M (2008) The dynamical response properties of neocortical neurons to temporally modulated noisy inputs in vitro. Cereb Cortex 18:2086-2097. 
König P, Engel AK, Singer W (1996) Integrator or coincidence detector? the role of the cortical neuron revisited. Trends Neurosci 19:130-137.

Kuchibhotla KV, Gill JV, Lindsay GW, Papadoyannis ES, Field RE, Sten TA, Miller KD, Froemke RC (2017) Parallel processing by cortical inhibition enables context-dependent behavior. Nat Neurosci 20:62-71.

Lee AT, Vogt D, Rubenstein JL, Sohal VS (2014) A class of GABAergic neurons in the prefrontal cortex sends long-range projections to the nucleus accumbens and elicits acute avoidance behavior. J Neurosci 34: $11519-11525$

Linaro D, Couto J, Giugliano M (2014) Command-line cellular electrophysiology for conventional and real-time closed-loop experiments. J Neurosci Methods 230:5-19.

Linaro D, Biró I, Giugliano M (2018) Dynamical response properties of neocortical neurons to conductance-driven time-varying inputs. Eur J Neurosci 47:17-32.

Litwin-Kumar A, Rosenbaum R, Doiron B (2016) Inhibitory stabilization and visual coding in cortical circuits with multiple interneuron subtypes. J Neurophysiol 115:1399-1409.

Litwin-Kumar A, Oswald AM, Urban NN, Doiron B (2011) Balanced synaptic input shapes the correlation between neural spike trains. PLoS Comput Biol 7:e1002305.

Ma Y, Hu H, Berrebi AS, Mathers PH, Agmon A (2006) Distinct subtypes of somatostatin-containing neocortical interneurons revealed in transgenic mice. J Neurosci 26:5069-5082.

Markram H, Toledo-Rodriguez M, Wang Y, Gupta A, Silberberg G, Wu C (2004) Interneurons of the neocortical inhibitory system. Nat Rev Neurosci 5:793-807.

Naud R, Gerstner W (2012) Coding and decoding with adapting neurons: a population approach to the peri-stimulus time histogram. PLoS Comput Biol 8:e1002711.

Ocker GK, Doiron B (2014) Kv7 channels regulate pairwise spiking covariability in health and disease. J Neurophysiol 112:340-352.

Ocker GK, Hu Y, Buice MA, Doiron B, Josić K, Rosenbaum R, Shea-Brown E (2017) From the statistics of connectivity to the statistics of spike times in neuronal networks. Curr Opin Neurobiol 46:109-119.

Packer AM, Yuste R (2011) Dense, unspecific connectivity of neocortical parvalbumin-positive interneurons: a canonical microcircuit for inhibition? J Neurosci 31:13260-13271.

Petilla Interneuron Nomenclature Group, Ascoli GA, Alonso-Nanclares L, Anderson SA, Barrionuevo G, Benavides-Piccione R, Burkhalter A, Buzsáki G, Cauli B, Defelipe J, Fairén A, Feldmeyer D, Fishell G, Fregnac Y, Freund TF, Gardner D, Gardner EP, Goldberg JH, Helmstaedter M, Hestrin S, et al. (2008) Petilla terminology: nomenclature of features of GABAergic interneurons of the cerebral cortex. Nat Rev Neurosci 9:557-568.

Pfeffer CK, Xue M, He M, Huang ZJ and Scanziani M (2013) Inhibition of inhibition in visual cortex: the logic of connections between molecularly distinct interneurons. Nat Neurosci 16:1068-1076
Poulet JF, Petersen CC (2008) Internal brain state regulates membrane potential synchrony in barrel cortex of behaving mice. Nature 454:881-885.

Press WH (2007) Numerical recipes, Ed. 3: The art of scientific computing. Cambridge: Cambridge University.

Richardson MJ (2009) Dynamics of populations and networks of neurons with voltage-activated and calcium-activated currents. Phys Rev E Stat Nonlin Soft Matter Phys 80:021928.

Robinson HP, Kawai N (1993) Injection of digitally synthesized synaptic conductance transients to measure the integrative properties of neurons. J Neurosci Methods 49:157-165.

Shea-Brown E, Josić K, de la Rocha J and Doiron B (2008) Correlation and synchrony transfer in integrate-and-fire neurons: basic properties and consequences for coding. Phys Rev Lett 100:108102.

Shinomoto S, Shima K, Tanji J (2003) Differences in spiking patterns among cortical neurons. Neural Comput 15:2823-2842.

Silberberg G, Markram H (2007) Disynaptic inhibition between neocortical pyramidal cells mediated by Martinotti cells. Neuron 53:735-746.

Sippy T, Yuste R (2013) Decorrelating action of inhibition in neocortical networks. J Neurosci 33:9813-9830.

Spruston N (2008) Pyramidal neurons: dendritic structure and synaptic integration. Nat Rev Neurosci 9:206-221.

Steinmetz PN, Manwani A, Koch C, London M, Segev I (2000) Subthreshold voltage noise due to channel fluctuations in active neuronal membranes. J Comput Neurosci 9:133-148.

Stuart GJ, Sakmann B (1994) Active propagation of somatic action potentials into neocortical pyramidal cell dendrites. Nature 367:69-72.

Tateno T, Robinson HP (2009) Integration of broadband conductance input in rat somatosensory cortical inhibitory interneurons: an inhibition-controlled switch between intrinsic and input-driven spiking in fast-spiking cells. J Neurophysiol 101:1056-1072.

Testa-Silva G, Verhoog MB, Linaro D, de Kock CP, Baayen JC, Meredith RM, De Zeeuw CI, Giugliano M, Mansvelder HD (2014) High bandwidth synaptic communication and frequency tracking in human neocortex. PLoS Biol 12:e1002007.

Tremblay R, Lee S, Rudy B (2016) GABAergic interneurons in the neocortex: from cellular properties to circuits. Neuron 91:260-292.

Tuckwell HC (1989) Stochastic processes in the neurosciences. Philadelphia (Pennsylvania): Society for Industrial and Applied Mathematics.

Uhlenbeck GE, Ornstein LS (1930) On the theory of the brownian motion. Phys Rev 36:823-841.

Wang Y, Gupta A, Toledo-Rodriguez M, Wu CZ, Markram H (2002) Anatomical, physiological, molecular and circuit properties of nest basket cells in the developing somatosensory cortex. Cereb Cortex 12:395-410.

Xu NL, Harnett MT, Williams SR, Huber D, O'Connor DH, Svoboda K, Magee JC (2012) Nonlinear dendritic integration of sensory and motor input during an active sensing task. Nature 492:247-251. 